\title{
Exponent Inequalities for the Bulk Conductivity of a Hierarchical Model
}

\section{K. Golden ${ }^{\star}$}

Department of Mathematics, Princeton University, Princeton, NJ 08544, USA

Received September 17, 1990; in revised form June 25, 1991

\begin{abstract}
The bulk conductivity $\sigma^{*}(p)$ of the bond lattice in $\mathbb{Z}^{d}$ is considered, where the bonds have conductivity 1 with probability $p$ or $\varepsilon \geqq 0$ with probability $1-p$. Various representations of the derivatives of $\sigma^{*}(p)$ are developed. These representations are used to analyze the behavior of $\sigma^{*}(p)$ for $\varepsilon=0$ near the percolation threshold $p_{c}$, when the conducting backbone is assumed to have a hierarchical node-link-blob (NLB) structure. This model has loops on arbitrarily many length scales and contains both singly and multiply connected bonds. Exact asymptotics of $\frac{d^{2} \sigma^{*}}{d p^{2}}$ for the NLB model are proven under some technical assumptions. The proof employs a novel technique whereby $\frac{d^{2} \sigma^{*}}{d p^{2}}$ for the NLB model with $\varepsilon=0$ and $p$ near $p_{c}$ is computed using perturbation theory for $\sigma^{*}(p)$ (for two- and threecomponent resistor lattices) around $p=1$ with a sequence of $\varepsilon$ 's converging to 1 as one goes deeper in the hierarchy. These asymptotics establish convexity of $\sigma^{*}(p)$ (for the NLB model) near $p_{c}$, and that its critical exponent $t$ obeys the inequalities $1 \leqq t \leqq 2$ for $d=2,3$, while $2 \leqq t \leqq 3$ for $d \geqq 4$. The upper bound $t=2$ in $d=3$, which is realizable in the NLB class, virtually coincides with two very recent numerical estimates obtained from simulation and series expansion for the original model.
\end{abstract}

\section{Introduction}

The transport properties of disordered media play an important role in many branches of science and engineering. For example, disordered conductors arise naturally in fields as varied as biology, geology, and solid state physics, yet also serve as key building blocks of advanced composite materials. Of particular

* Supported in part by NSF Grant DMS-8801673 and AFOSR Grant AFOSR-90-0203

Present address: Department of Mathematics, University of Utah, Salt Lake City, UT 84112, USA 
practical and theoretical interest are those systems which undergo an insulator/conductor transition as some parameter is varied. The simplest model which exhibits this complex macroscopic behavior is the random resistor network [1-3] based on the percolation model [4]. Introduced in the late 1960's as a model for impure semiconductors, this network is very simply defined, yet displays the above type of phase transition, and thus occupies a central place in the theory of disordered conductors.

In particular, consider the bulk conductivity $\sigma^{*}(p)$ of the bond lattice in $\mathbb{Z}^{d}$, where the conductivity of the bonds is either 1 with probability $p$, or $\varepsilon \geqq 0$ with probability $1-p$. When $\varepsilon=0$, we can view the conductivity 0 bonds as vacant. In this case, the underlying percolation problem concerns $P_{\infty}(p)$, the probability that the origin is connected to an infinite cluster of occupied (conductivity 1) bonds. For $p$ below some critical probability $p_{c}$, called the percolation threshold, $P_{\infty}(p)=0$, while for $p>p_{c}, P_{\infty}(p)>0$. The bulk conductivity $\sigma^{*}(p)$ has a similar behavior, with $\sigma^{*}(p)=0$ for $p<p_{c}$, since there can be no conduction through the lattice if there are no infinite pathways of occupied bonds, and $\sigma^{*}(p)>0$ for $p>p_{c}$, although there is apparently no simple relation between $\sigma^{*}(p)$ and $P_{\infty}(p)$ [5]. As $p \rightarrow p_{c}^{+}$, it is believed that $\sigma^{*}(p)$ exhibits critical scaling, $\sigma^{*}(p) \sim\left(p-p_{c}\right)^{t}$, where $t$ is called the conductivity critical exponent [6].

The pure percolation problem has been studied extensively in both the physics [7] and mathematics literature [8,9]. However, the random resistor network, which has been widely studied in the physics literature $[10,11]$, has received comparatively little attention in the mathematics community. The main exceptions are the works of Grimmett and Kesten [12] (see also [8]), Chayes and Chayes [13], and Kozlov [14], who considers similar problems in the continuum. One of the principal contributions of the first two works is to establish the coincidence of the conduction and percolation thresholds for $d=2$, where $p_{c}=\frac{1}{2}[12]$, as well as for higher dimensions [13]. In addition, the Chayes [13] obtain some bounds on $\sigma^{*}(p)$ in terms of percolation quantities, such as $\sigma^{*}(p) \leqq p d\left[P_{\infty}(p)\right]^{2}$, which yield bounds on the critical exponent $t$ (assuming it exists) in terms of percolation exponents. It has also been established (rigorously in $d=2$ ) that $t \geqq 1[15,16]$.

While the above described works on the random resistor network have focussed on relating $\sigma^{*}(p)$ to percolation quantities, here we concentrate on obtaining information about $\sigma^{*}(p)$ directly through analysis of its derivatives with respect to $p$, particularly the second derivative. The idea behind analyzing the derivatives is that qualitative information, such as the vanishing or diverging as $p \rightarrow p_{c}^{+}$of the first, second, and third derivatives of $\sigma^{*}(p)$, which have exponents $t-1, t-2$, and $t-3$, respectively, gives the relation of $t$ to the numbers 1,2 , and 3 , rather than to other unknown critical exponents. This approach was initially motivated by the simple observation that in numerical simulations $[6,17-20]$ of the graph of $\sigma^{*}(p)$ for bond or site models in $d \geqq 2$, it appears that $\sigma^{*}(p)$ is convex, i.e., $\frac{d^{2} \sigma^{*}}{d p^{2}} \geqq 0$, at least for $p$ near $p_{c}$. Our investigation [21] of this convexity led to the current analysis.

Before we describe our main results, it is necessary to discuss in what context they are obtained. As evidenced by the lack of substantial mathematical progress on the bulk conductivity problem, a rigorous understanding of $\sigma^{*}(p)$ when $\varepsilon=0$ is a formidable problem. For example, it is not even known rigorously if $\sigma^{*}(p)$ has derivatives when $\varepsilon=0$, although there is current progress in this direction $[14,22]$ 
in the continuum, and we have proven that $\sigma^{*}(p)$ is analytic in $p$ for any $\varepsilon>0$. Nevertheless, for finite volumes, where $\sigma^{*}(p)$ for $\varepsilon=0$ is a polynomial, we can obtain rigorous representations of its derivatives involving the conductivities of backbone configurations with appropriate numbers of bonds removed (the backbone is the subset of occupied bonds which excludes dangling bonds where no current flows). The first assumption under which our results are obtained is the existence of the infinite volume limits of these derivatives, up to, say, third order.

The second assumption is more serious. As stated above, our derivative representations involve complex calculations on the backbone. However, the backbone is a very complicated object whose structure is by no means fully understood. In order to make progress, we assume that the backbone near $p_{c}$ has a hierarchical node-link-blob (NLB) structure, which is based on the works of Stanley [23] and Coniglio [24]. This model consists of a "super-lattice" of beaded necklaces (links and blobs) connecting nodes, which are separated by a correlation length $\xi$; see Fig. 1 . The bonds in the links (strings) are singly connected - removal of a single one breaks a connection between nodes, and the bonds in the blobs (beads) are multiply connected. The blobs have a hierarchical structure, being composed themselves of links and blobs in a self-similar fashion. This model then has loops on arbitrarily many length scales, and reflects what is known rigorously $[13,24]$ and heuristically about the structure of the backbone. It is for this model that our main results about the critical exponent $t$ are obtained.

We now describe the results. First we obtain representations of the derivatives of $\sigma^{*}(p)$ valid for any $\varepsilon \geqq 0$ (assuming existence for $\varepsilon=0$ at infinite volume). We then derive rigorous perturbation expansions around a homogeneous medium $(\varepsilon=1)$ of the derivatives of $\sigma^{*}(p)$ (for infinite volume) which will be used in the calculation on the NLB model. A key step here and later will be the analyticity of $\sigma^{*}(p)$ for $\varepsilon>0$. In addition, we shall prove analogous formulas and an analyticity result for the conductivity of the bond lattice with three different conductivities randomly assigned to the bonds, which will play an interesting role in the NLB calculation. Then we give numerical and rigorous results outlining the regimes in $\varepsilon$ and $p$ of convexity of $\sigma^{*}(p)$. The main results here are that in $d=2$, while $\sigma^{*}(p)$ cannot be convex for all $p$ when $\varepsilon=0$, it is convex for any $\varepsilon>0$ in a neighborhood containing $p_{c}=\frac{1}{2}$. Then, as a preliminary to the NLB calculation, we investigate the geometry of convexity, i.e., how different pairings of bonds in a graph contribute to the overall sign of the second derivative of the conductivity. Finally, using our representations, we obtain exact asymptotics $\frac{d^{2} \sigma^{*}}{d p^{2}}$ as $p \rightarrow p_{c}^{+}$for the NLB model. The calculation yields not only convexity near $p_{c}$ (under a numerically verified assumption concerning $\frac{d^{2} \sigma^{*}}{d p^{2}}$ at $\left.p=1\right)$ which implies $t \geqq 1$, but delineates in which dimensions $\frac{d^{2} \sigma^{*}}{d p^{2}} \rightarrow 0,+\infty$, or a positive constant as $p \rightarrow p_{c}^{+}$. Combining this information with the scaling law $\frac{d^{2} \sigma^{*}}{d p^{2}} \sim\left(p-p_{c}\right)^{t-2}$ yields the inequalities $1 \leqq t \leqq 2$ for $d=2,3$ and $2 \leqq t \leqq 3$ for $d \geqq 4$. The inequality $t \leqq 3$ for $d \geqq 4$ is obtained by applying a similar analysis to $\frac{d^{3} \sigma^{*}}{d p^{3}}$, and can be viewed as a mean-field bound, since it is believed that $t=3$ for $d \geqq 6$. In this case we analyze only the simpler node-link model, without the blobs, as it is believed to be adequate in higher dimensions. For 
clarity, we remark that the convexity and exponent inequalities are not rigorous for the actual backbone near $p_{c}$ for the original lattice, but are rigorous for the NLB model of the backbone, under the technical assumptions about the derivatives. However, we believe that this model adequately reflects the relevant features of the actual backbone, so that presumably our inequalities hold for the original lattice.

One aspect of our NLB calculation that we would like to stress is the following. The NLB model can be viewed as a base lattice $G$ whose "bonds" consist of the above described hierarchical necklaces. Our representation of $\frac{d^{2} \sigma^{*}}{d p^{2}}$ involves lattice sums of second differences of the conductivities of the model, where the sum is over all possible pairs of bonds in the model, and for any given pair the second difference involves removal of all combinations of bonds in the pair. Because at most two bonds are removed at a time, it is possible to compute the sums via perturbation around $p=1$ of the derivatives of the bulk conductivity of the base lattice $G$. Furthermore, as $p$ gets close to $p_{c}$, the removal of any multiply connected bond has very little effect on the conductivity of that necklace. Thus the difference between the conductivity of that necklace and the conductivity of all the other necklaces in the model with no bonds removed is very slight, so that it is possible to use perturbation around a homogeneous medium of the derivatives of the bulk conductivity of the base lattice $G$. One complication that must be dealt with is when one bond removed is in one necklace, and the other bond is in another necklace but at a different level of the hierarchy. In this case the base lattice $G$ can be viewed as having bonds of three different types (the two above and all the other necklaces with no removals), and it is then necessary to use perturbation theory for resistor lattices with three components, which we develop here. Overall then, what is accomplished is that through the hierarchical model, we obtain information about $\sigma^{*}(p)$ and its derivatives with $\varepsilon=0$ and $p$ near $p_{c}$ via perturbation theory around $p=1$ with a sequence of $\varepsilon$ 's converging to 1 . It is important to remark further that we have been forced to develop these techniques for analyzing $\sigma^{*}(p)$ and its derivatives because simply taking the $\varepsilon \rightarrow 0$ limit of the above described perturbation expansions or of classical bounds on $\sigma^{*}(p)$ (such as the harmonic and arithmetic or Hashin-Shtrikman bounds) gives essentially no information in the $\varepsilon=0$ case.

Another point that should be made is that the dimensional dependence of our NLB model is through the macroscopic lattice structure. The microscopic structure of the multiply connected blobs in the necklaces is assumed to be independent of dimension for computational simplicity. However, one of the main points of our calculation is that the dominant term in the asymptotics of $\frac{d^{2} \sigma^{*}}{d p^{2}}$ for the NLB model comes from singly connected bonds, and that changing the details of the blobs (such as according to dimension) only changes the constants in higher order corrections. For this reason we expect that the present calculation might serve as the basis of a rigorous calculation on the actual backbone.

Our results for $d=3$ are particularly intriguing. First, the inequality $t \leqq 2$ excludes roughly one fourth of published numerical estimates of $t$ in $d=3$, which have ranged from 1.5 to 2.36. Furthermore, this inequality is based on an exact calculation of $t=2$ for one particular NLB model which provides an upper bound on $t$ for the full class of NLB models (indexed by the length of the "beaded necklaces" connecting the nodes, relative to the correlation length separating the 
nodes). In view of this result, it is quite striking that very recently, Gingold and Lobb [25] have obtained for $d=3$ the numerical estimate $t=2.003 \pm 0.047$, which is based on the application of finite size scaling techniques to conductivity data from lattices of size up to $(80)^{3}$, and Alder et al. [26] have obtained the estimate $t=2.02 \pm 0.05$ from a $13^{\text {th }}$ order series expansion of the resistive susceptibility. In addition, our inequality is compatible with the results of an $\varepsilon=6-d$ expansion [27], and the general view that "roughly $t=2$ " [7]. (We should also mention the recent work of Roman [28] on the ant-in-the-labyrinth problem, who indirectly obtains a value of $t \approx 2.16$. However, he acknowledges the inconsistency with other results, which is discussed in [25].) The recent numerical results in [25] and [26], in conjunction with our rigorous calculations on the NLB model, suggest the possibility that $t=2$ is an exact result for $d=3$. To our knowledge, the present work is the first to relate $t$ in a direct and natural way to the number 2, rather than to other (unknown) critical exponents of percolation theory previously mentioned.

The results of this paper have already been announced in [29].

\section{Formulation}

We give two formulations of the conductivity problem, both of which will be used in subsequent sections. The first formulation is for an arbitrary graph. The second is for the bond lattice, which is a special case of a stationary random medium.

Let $G$ be a finite graph consisting of $N$ bonds $\left\{b_{i}\right\}$ and $M$ vertices $\left\{x_{i}\right\}$. Assigned to $G$ are $N$ independent random variables $c_{i}, 1 \leqq i \leqq N$, the bond conductivities, which take the values 1 with probability $p$ and $\varepsilon \geqq 0$ with probability $q=1-p$. Distinguish two vertices, say $x_{1}=x$ and $x_{M}=y$, and connect them to a battery which keeps the voltage drop between them equal to 1 . The effective conductivity $\tilde{\sigma}(\omega)$ of the network for any realization $\omega$ of the bond conductivities is just the total current $i(\omega)$ that flows through the network, which is obtained via Kirchoff's laws. We define $\sigma(p)=\langle\tilde{\sigma}(\omega)\rangle$, where the expectation $\langle\cdot\rangle$ is over all $2^{N}$ realizations. For example, a two bond network has

$$
\sigma(p)=p^{2} \tilde{\sigma}(1,1)+p q(\tilde{\sigma}(1, \varepsilon)+\tilde{\sigma}(\varepsilon, 1))+q^{2} \tilde{\sigma}(\varepsilon, \varepsilon),
$$

where $\tilde{\sigma}(1,1)=\tilde{\sigma}(\omega)$ with $\omega=(1,1)$, and so on. For $N$ bonds, $\sigma(p)$ is an $N^{\text {th }}$ order homogeneous polynomial in $p$ and $q$,

$$
\begin{gathered}
\sigma(p)=\sum_{k=0}^{N} \alpha_{k} p^{N-k} q^{k}, \\
\alpha_{k}=\sum_{\omega^{k} \in \Omega^{k}} \tilde{\sigma}\left(\omega^{k}\right), \quad q=1-p,
\end{gathered}
$$

where $\Omega^{k}=\left\{\omega^{k}=\left(\omega_{1}, \ldots, \omega_{N}\right) \mid \omega_{l}=\varepsilon\right.$ for exactly $k$ of the $\omega_{l}$ 's $\}$.

The cases of most interest are when $G$ is a square, cubic, or hypercubic lattice. Then, with $d=2$ for simplicity, we take an $L \times L$ sample of the lattice and attach a perfectly conducting bus bar to each of two opposite edges of the sample. This can be accomplished [12] in the above language by attaching to each vertex of these opposing edges a perfectly conducting bond. All of these bonds from one edge meet at a new vertex $x$ and all the bonds from the other edge meet at a new vertex $y$. Then $x$ and $y$ are connected again with the unit battery. Random bond conductivities are assigned only to the bonds in the original $L \times L$ sample. Let $\sigma_{L}(p)$ denote (2.1) for 
the effective conductivity measured between $x$ and $y$. Then for $d \geqq 1$, the finite volume bulk conductivity $\sigma_{L}^{*}(p)$ is defined as

$$
\sigma_{L}^{*}(p)=L^{2-d} \sigma_{L}(p)
$$

Finally, we define the bulk conductivity $\sigma^{*}(p)$ by

$$
\sigma^{*}(p)=\lim _{L \rightarrow \infty} \sigma_{L}^{*}(p)
$$

For $\varepsilon>0$, the infinite volume limit in (2.3) has been shown to exist [30-33], and for $\varepsilon=0$ the existence of $\sigma^{*}$ has recently been proven in the continuum [34], but the methods employed extend to the discrete situation considered here.

We now formulate the bulk conductivity problem for the bond lattice, as a special case of the stationary random media considered in $[31,32]$. Let $(\Omega, P)$ be a probability space, and let $\sigma^{i}(x, \omega)$ be a stationary stochastic process in $x \in \mathbb{Z}^{d}$ and $\omega \in \Omega$, taking the values $\sigma_{1}$ and $\sigma_{2}$ on the bond emanating from $x$ in the positive $i^{\text {th }}$ direction, $i=1, \ldots, d$, with probabilities $1-p$ and $p$ (we shall subsequently take $\sigma_{1}=\varepsilon$ and $\sigma_{2}=1$ ). The space $\Omega$ represents the set of all realizations of the random medium, and can be identified with $\left\{\sigma_{1}, \sigma_{2}\right\}^{d \mathbb{Z}^{d}}$. The measure $P$ is compatible with the stationarity, i.e., it is invariant under the translation group $\tau_{y}: \Omega \rightarrow \Omega$ defined by $\tau_{y} \omega(x)=\omega(x+y), \forall x, y \in \mathbb{Z}^{d}, \omega \in \Omega$. Since we consider here two component media, $\sigma^{i}(x, \omega)$ can be written as

$$
\sigma^{i}(x, \omega)=\sigma_{1} \chi_{1}^{i}(x, \omega)+\sigma_{2} \chi_{2}^{i}(x, \omega), \quad i=1, \ldots, d,
$$

where the characteristic function $\chi_{j}^{i}(x, \omega)$ equals one for all realizations $\omega$ which have medium $j$ in the $i^{\text {th }}$ positive bond at $x, j=1,2, i=1, \ldots, d$, and equals zero otherwise. Let $E^{k}(x, \omega), J^{k}(x, \omega) \in L^{2}(\Omega, P)$ be the stationary random electric and current fields satisfying

$$
\begin{gathered}
J_{i}^{k}(x, \omega)=\sigma^{i}(x, \omega) E_{i}^{k}(x, \omega), \quad i=1, \ldots, d, \\
\sum_{i=1}^{d} D_{i}^{-} J_{i}^{k}(x, \omega)=0, \\
D_{i}^{+} E_{j}^{k}(x, \omega)-D_{j}^{+} E_{i}^{k}(x, \omega)=0, \quad i, j=1, \ldots, d, \\
\int_{\Omega} P(d \omega) E^{k}(x, \omega)=e_{k},
\end{gathered}
$$

where $e_{k}$ is a unit vector in the $k^{\text {th }}$ direction. In (2.6) and (2.7), $D_{i}^{+}$and $D_{i}^{-}$are the forward and backward difference operators

$$
D_{i}^{+}=T_{i}^{+}-I, \quad D_{i}^{-}=I-T_{i}^{-}, \quad i=1, \ldots, d,
$$

where $I$ is the identity, and the $T_{i}^{+}=T_{+e_{i}}$ and $T_{i}^{-}=T_{-e_{i}}$ are the generators (through composition) of the unitary group $T_{x}$ acting on $L^{2}(\Omega, P)$ defined by $\left(T_{x} f\right)(\omega)=f\left(\tau_{x} \omega\right)=f(x, \omega)$ for any $f \in L^{2}(\Omega, P)$, which is a stationary process on $\mathbb{Z}^{d}$ and $\Omega$. By stationarity, we may now focus attention at $x=0$, and subsequently drop the $x$-notation.

The bulk conductivity tensor $\sigma_{j k}^{*}$ may now be defined as

$$
\sigma_{j k}^{*}=\int_{\Omega} P(d \omega) \sigma^{j}(\omega) E_{j}^{k}(\omega)
$$


Since the distribution of bond conductivities in (2.4) for our problem is independent of $i$, the medium is isotopic, i.e., $\sigma_{j k}^{*}=\sigma^{*} \delta_{j k}$. We thus focus on one diagonal coefficient, $\sigma^{*}=\sigma_{k k^{*}}$. Furthermore, since our equations are linear in $\sigma^{k}(\omega)$, $\sigma^{*}$ depends only on the ratio $\sigma_{1} / \sigma_{2}$, that is, $\sigma^{*}$ is homogeneous of degree one in the $\sigma_{i}$ 's. Thus it suffices to let $\sigma_{1}=\varepsilon$ and $\sigma_{2}=1$. We then define

$$
m(\varepsilon)=\sigma^{*}=\sigma_{k k}^{*}=\int_{\Omega} P(d \omega)\left(\varepsilon \chi_{1}(\omega)+\chi_{2}(\omega)\right) E_{k}^{k}(\omega),
$$

where for simplicity we have now dropped the " $k$ " superscript from $\chi_{j}$ (due to isotropy of distribution). It has been proven $[32,35]$ that $m(\varepsilon)$ is analytic off the negative real axis $(-\infty, 0]$ in the $\varepsilon$-plane, and that $m$ maps the upper half plane to the upper half plane, i.e., $\operatorname{Im}(m(\varepsilon))>0$ when $\operatorname{Im}(\varepsilon)>0$. It is useful to introduce the new function $F(s)=1-m(\varepsilon), s=1 /(1-\varepsilon)$, which is analytic off $[0,1]$ in the $s$-plane. In [32] it was proven that $F(s)$ has the integral representation

$$
F(s)=1-m(\varepsilon)=\int_{0}^{1} \frac{d \mu(y)}{s-y}, \quad s=1 /(1-\varepsilon) \notin[0,1],
$$

where $\mu$ is a positive Borel measure on $[0,1]$. This representation can be proven either as a consequence of the Herglotz Theorem in analytic function theory [36], or as a consequence of the spectral theorem applied to the operator representation [32] of $F(s)$ arising from (2.5)-(2.8),

$$
F(s)=\int_{\Omega} P(d \omega) \chi_{1}(\omega)\left[\left(s+\Gamma \chi_{1}\right)^{-1} \varepsilon_{k}\right] \cdot e_{k},
$$

where $\Gamma=\nabla^{+}(-\Delta)^{-1} \nabla^{-},, \nabla^{ \pm}=\left(D_{1}^{ \pm}, \ldots, D_{d}^{ \pm}\right)$, and $(-\Delta)^{-1}$ is the inverse of the lattice Laplacian

$$
\Delta=\sum_{i=1}^{d} D_{i}^{-} D_{i}^{+} .
$$

In the Hilbert space $L^{2}(\Omega, P)$ with weight $\chi_{1}$ in the inner product, $\Gamma \chi_{1}$ is a bounded self adjoint operator with norm less than or equal to 1 . The formula (2.12) is the spectral representation of the resolvent $\left(s+\Gamma \chi_{1}\right)^{-1}$, where $\mu$ is the spectral measure of the family of projections of $\Gamma \chi_{1}$ in the state $e_{k}$.

\section{Representations of the Derivatives of the Bulk Conductivity}

\subsection{Two-Component Media}

In this section we shall derive the representations of the derivatives of $\sigma^{*}(p)$ which will be used extensively in Sect. 4 and 5. First we begin with an arbitrary graph $G$ with $N$ bonds having conductivities 1 or $\varepsilon \geqq 0$, as in the beginning of Sect. 2. Let $\omega^{k}$ be as in (2.1). Now define

$$
\delta \tilde{\sigma}\left(\omega^{k}\right)=\sum_{i=1}^{N-k}\left[\tilde{\sigma}\left(\omega_{i}^{k}(1)\right)-\tilde{\sigma}\left(\omega_{i}^{k}(\varepsilon)\right)\right],
$$

where given $\omega^{k}, i$ runs over the $N-k$ bonds which have conductivity $1, \omega_{i}^{k}(1)=\omega^{k}$, and $\omega_{i}^{k}(\varepsilon)$ is the same realization but with the $i^{\text {th }}$ bond conductivity changed to $\varepsilon$. Similarly, let

$$
\delta^{2} \tilde{\sigma}\left(\omega^{k}\right)=\sum_{\substack{i, j=1 \\ i \neq j}}^{N-k}\left[\tilde{\sigma}\left(\omega_{i j}^{k}(1,1)\right)+\tilde{\sigma}\left(\omega_{i j}^{k}(\varepsilon, \varepsilon)\right)-\tilde{\sigma}\left(\omega_{i j}^{k}(1, \varepsilon)\right)-\tilde{\sigma}\left(\omega_{i j}^{k}(\varepsilon, 1)\right)\right],
$$


where, given $\omega^{k}, i$ and $j$ run over the $N-k$ bonds which have conductivity 1 , $\omega_{i j}^{k}(1,1)=\omega^{k}, \omega_{i j}^{k}(\varepsilon, \varepsilon)$ is $\omega^{k}$ but with the $i^{\text {th }}$ and $j^{\text {th }}$ bond conductivities changed to $\varepsilon$, $\omega_{i j}^{k}(1, \varepsilon)$ is $\omega^{k}$ but with the $j^{\text {th }}$ bond conductivity changed to $\varepsilon$, and similarly for $\omega_{i j}^{k}(\varepsilon, 1)$ with $i$ instead of $j$. The expressions in (3.1) and (3.2) represent discrete first and second derivatives of the conductivity with respect to $p$. To make this connection more precise, we define

$$
\begin{aligned}
& \beta_{k}=\sum_{\omega^{k} \in \Omega^{k}} \delta \tilde{\sigma}\left(\omega^{k}\right), \\
& \gamma_{k}=\sum_{\omega^{k} \in \Omega^{k}} \delta^{2} \tilde{\sigma}\left(\omega^{k}\right) .
\end{aligned}
$$

Then the exact relation is contained in

Lemma 3.1. Let $G$ be any $N$-bond graph with bond conductivities 1 and $\varepsilon \geqq 0$. Then

$$
\begin{aligned}
& \frac{d \sigma}{d p}=\sum_{k=0}^{N-1} \beta_{k} p^{N-1-k} q^{k}, \\
& \frac{d^{2} \sigma}{d p^{2}}=\sum_{k=0}^{N-2} \gamma_{k} p^{N-2-k} q^{k} .
\end{aligned}
$$
Proof. Differentiate $\sigma(p)$ in (2.1) keeping in mind that since $q=1-p, \frac{d}{d p}=-\frac{d}{d q}$.
Then we have

$$
\begin{aligned}
\frac{d \sigma}{d p} & =\sum_{k=0}^{N} \alpha_{k}\left[(N-k) p^{N-k-1} q^{k}-k p^{N-k} q^{k-1}\right] \\
& =\sum_{k=0}^{N-1} p^{N-k-1} q^{k}\left[(N-k) \alpha_{k}-(k+1) \alpha_{k+1}\right]
\end{aligned}
$$

which can be written as (3.5). Taking one more derivative, we have

$$
\begin{aligned}
\frac{d^{2} \sigma}{d p^{2}}= & \sum_{k=0}^{N} \alpha_{k}\left[(N-k)(N-k-1) p^{N-k-2} q^{k}\right. \\
& \left.-2(N-k) k p^{N-k-1} q^{k-1}+k(k-1) p^{N-k} q^{k-2}\right] \\
= & \sum_{k=0}^{N-2} p^{N-2-k} q^{k}\left[(N-k)(N-k-1) \alpha_{k}\right. \\
& \left.-2(N-k-1)(k+1) \alpha_{k+1}+(k+2)(k+1) \alpha_{k+2}\right],
\end{aligned}
$$

which can be written as (3.6).

When the number of bonds in $G$ goes to infinity, the appropriate limits of $\beta_{k}$ and $\gamma_{k}$ yield exact formulas for the first and second derivatives of $\sigma(p)$. When $G$ is a hypercubic sample of the lattice with side $L$, then $N \sim d L^{d}$. In this case we consider $\sigma^{*}(p)$ in (2.3), and assume for $\varepsilon=0$ that the infinite volume limits of the derivatives exist for $p>p_{c}$ (existence for all $p$ when $\varepsilon>0$ is contained in a subsequent theorem). Then we have

Lemma 3.2. For the lattice in $d \geqq 2$ with $\varepsilon \geqq 0$ and fixed $p$,

$$
\begin{gathered}
p \frac{d \sigma^{*}}{d p}=\lim _{k, L \rightarrow \infty} L^{2-d} \beta_{k} /\left(\frac{N}{k}\right), \\
p^{2} \frac{d^{2} \sigma^{*}}{d p^{2}}=\lim _{k, L \rightarrow \infty} L^{2-d} \gamma_{k} /\left(\frac{N}{k}\right),
\end{gathered}
$$


where the simultaneous limits of $k$ and $L \rightarrow \infty$ are taken so that

$$
\lim _{k, L \rightarrow \infty} \frac{k}{N}=\lim _{k, L \rightarrow \infty} \frac{k}{d L^{d}}=q=1-p .
$$

Proof. Equation (3.5) can be written as

$$
p \frac{d \sigma}{d p}=\sum_{k=0}^{N-1} \beta_{k} p^{N-k} q^{k}
$$

Note that in (3.3) there are $\left(\begin{array}{l}N \\ k\end{array}\right)$ terms $\delta \tilde{\sigma}\left(\omega^{k}\right)$. When $N$ is large, the weight of the binomial distribution is concentrated on values of $k$ such that $k / N$ is nearly $q=1-p$. Appropriately scaling $\beta_{k}$ with $L^{2-d}$ yields the result (3.11), and similarly for (3.12). See [37] for more details about this type of argument.

When $\varepsilon=0$ formulas (3.11) and (3.12) take a particularly nice form. In (3.1) and (3.2), any bonds of $\omega^{k}$ of conductivity 1 which have no current running through them do not contribute to the sums. These bonds are called dangling bonds. The set of bonds which do have current in them is called the backbone. Let $B(p)$ denote a backbone configuration for fixed $p$. Then the right-hand sides of (3.11) and (3.12) can be written as $\delta \sigma^{*}(B(p))$ and $\delta^{2} \sigma^{*}(B(p))$, which are the scaled infinite volume limits of (3.3) and (3.4) with $\omega^{k}$ replaced by $B(p)$, averaged over realizations of the backbone for fixed $p$. We summarize this observation in

Theorem 3.1. For the lattice in $d \geqq 2$ with $\varepsilon=0$ and fixed $p$,

$$
\begin{aligned}
p \frac{d \sigma^{*}}{d p} & =\delta \sigma^{*}(B(p)), \\
p^{2} \frac{d^{2} \sigma^{*}}{d p^{2}} & =\delta^{2} \sigma^{*}(B(p)),
\end{aligned}
$$

where the right-hand sides in (3.15) and (3.16) are averaged over backbone configurations $B(p)$.

In order to derive the perturbation expansions for the derivatives of $\sigma^{*}(p)$ for $\varepsilon>0$ we shall require the following analyticity result, which was originally proved in [21], and will only be stated here.

Theorem 3.2. $(d \geqq 1)$ For every $\varepsilon>0$, there exists an open neighborhood $V_{\varepsilon}$ in the complex p-plane such that $[0,1] \subset V_{\varepsilon}$ and $\sigma^{*}(p)$ is analytic in $V_{\varepsilon}$.

The idea of the proof is to produce a neighborhood containing $[0,1]$ in which a subsequent perturbation expansion (3.17) of $\sigma^{*}(p, s)$ converges uniformly.

Remark 1. Theorem 3.2 and its proof hold for a large class of continuum systems as well, namely infinitely interchangeable media, which have recently been introduced by Bruno [38]. This class is a generalization of Miller's cell materials [39], where all of space is divided up into cells which are randomly assigned two conductivities with probabilities $p$ and $1-p$. The details are contained in [40] [along with rigorous upper and lower bounds on $\sigma^{*}(p)$ for the $d=2$ bond problem with $\varepsilon>0$ ]. 
Remark 2. Theorem 3.2 and its generalization to continuum systems can be viewed as a Lee-Yang Theorem: for $\varepsilon>0, \sigma^{*}(p, \varepsilon)$ has no phase transition in $p$, i.e., it is analytic in $p$. This is an analogue of the statement that for positive magnetic field, the free energy for an Ising model has no phase transition in temperature T, i.e., it is analytic in $T$. The natural question arises, then, as to how analyticity of $\sigma^{*}(p)$ is lost as $\varepsilon \rightarrow 0$, i.e., how does the phase transition arise? Is it due to a pinching of zeros in the complex $p$-plane around $p_{c}$, as in a Lee-Yang picture? This question is adressed numerically in [41].

Let us now expand (2.12) for $|s|>1$ around a homogeneous medium $(s=\infty$ or $\varepsilon=1)$ to obtain

$$
\begin{gathered}
1-\sigma^{*}(p, s)=\frac{\mu_{0}(p)}{s}+\frac{\mu_{1}(p)}{s^{2}}+\frac{\mu_{2}(p)}{s^{3}}+\ldots, \\
\mu_{n}=\int_{0}^{1} y^{n} d \mu(y) .
\end{gathered}
$$

By expanding (2.13) similarly and equating coefficients, we obtain

$$
\mu_{n}=(-1)^{n} \int_{\Omega} P(d \omega)\left[\chi_{1}\left(\Gamma \chi_{1}\right)^{n} e_{k}\right] \cdot e_{k},
$$

for any $k=1, \ldots, d$. Clearly, $\mu_{0}=1-p$, and the discrete analog of a calculation in [32] yields $\mu_{1}=p(1-p) / d$.

Thus (3.17) to second order is

$$
1-\sigma^{*}(p, s)=\frac{1-p}{s}+\frac{p(1-p) / d}{s^{2}}+\ldots
$$

We remark that in $d=2$, using arguments of infinite interchangeability, in [40] we calculated two more moments of $\mu$ to obtain

$$
\mu_{2}=\frac{p(1-p)}{4}, \quad \mu_{3}=\frac{p(1-p)(1+p(1-p))}{8} .
$$

Now, using the analyticity of $\sigma^{*}(p)$ for $\varepsilon>0$, we can immediately differentiate (3.20) to obtain

Theorem 3.3. For the hypercubic lattice in $d \geqq 1$ with $\varepsilon>0$, the derivatives of $\sigma^{*}(p)$ have the following expansions, where $s=1 /(1-\varepsilon)$,

$$
\begin{gathered}
\frac{d \sigma^{*}}{d p}=\frac{1}{s}+\frac{(2 p-1) / d}{s^{2}}+O\left(\frac{1}{s^{3}}\right), \\
\frac{d^{2} \sigma^{*}}{d p^{2}}=\frac{2 / d}{s^{2}}+O\left(\frac{1}{s^{3}}\right) .
\end{gathered}
$$

Furthermore, for $d=2$, we have

$$
\frac{d^{2} \sigma^{*}}{d p^{2}}=\frac{1}{s^{2}}+\frac{1 / 2}{s^{3}}+\frac{\frac{3}{2} p(1-p)}{s^{4}}+O\left(\frac{1}{s^{5}}\right) .
$$




\subsection{Three-Component Media}

In the calculation of $\frac{d^{2} \sigma^{*}}{d p^{2}}$ for the NLB model we shall require some representations of the derivatives of $\sigma^{*}$ for three-component media. We begin by briefly formulating this problem and giving definitions analogous to (2.1).

Let $G$ be any graph consisting of $N$ bonds. We randomly assign to the bonds the conductivities 1 with probability $p, \varepsilon_{1}$ with probability $q_{1}$, or $\varepsilon_{2}$ with probability $q_{2}$, so that $p=1-q_{1}-q_{2}$. Again we distinguish two vertices and measure the conductivity of the network between these two vertices, and average this conductivity over all $3^{N}$ realizations. Analogous to (2.1) we define

$$
\begin{aligned}
\sigma(p) & =\sum_{\substack{k, m=0 \\
k+m \leqq N}}^{N} \alpha_{k m} p^{N-k-m} q_{1}^{k} q_{2}^{m}, \\
\alpha_{k m} & =\sum_{\omega^{k m} \in \Omega^{k m}} \tilde{\sigma}\left(\omega^{k m}\right),
\end{aligned}
$$

where $\Omega^{k m}=\left\{\omega^{k m}=\left(\omega_{1}, \ldots, \omega_{N}\right) \mid \omega_{l}=\varepsilon_{1}\right.$ for exactly $k$ of the $\omega_{l}$ 's and $\omega_{l}=\varepsilon_{2}$ for exactly $m$ of the $\left.\omega_{l}^{\prime} s\right\}$.

Our use of the derivatives of $\sigma^{*}$ for three-component media will be restricted to the second mixed partial with respect to $q_{1}$ and $q_{2}$. To this end, analogous to (3.2), we define

$$
\begin{aligned}
\delta_{12}^{2} \tilde{\sigma}\left(\omega^{k m}\right)= & \sum_{\substack{i, j=1 \\
i \neq j}}^{N-k-m}\left[\tilde{\sigma}\left(\omega_{i j}^{k m}(1,1)\right)+\tilde{\sigma}\left(\omega_{i j}^{k m}\left(\varepsilon_{1}, \varepsilon_{2}\right)\right)\right. \\
& \left.-\tilde{\sigma}\left(\omega_{i j}^{k m}\left(1, \varepsilon_{1}\right)\right)-\tilde{\sigma}\left(\omega_{i j}^{k m}\left(1, \varepsilon_{2}\right)\right)\right],
\end{aligned}
$$

which is a discrete version of this mixed partial, where $\omega_{i j}^{k m}(1,1)=\omega_{i j}^{k m}, \omega_{i j}^{k m}\left(\varepsilon_{1}, \varepsilon_{2}\right)$ is $\omega_{i j}^{k m}$ with conductivity $\varepsilon_{1}$ for the $i^{\text {th }}$ bond and conductivity $\varepsilon_{2}$ for the $j^{\text {th }}$ bond, and so on. Now define

$$
v_{k m}=\sum_{\omega^{k m} \in \Omega^{k m}} \delta_{12}^{2} \tilde{\sigma}\left(\omega^{k m}\right) .
$$

Then we state the following lemma, whose proof is similar to that for Lemma 3.1.

Lemma 3.3. Let $G$ be any $N$ bond graph with conductivities $1, \varepsilon_{1} \geqq 0$ and $\varepsilon_{2} \geqq 0$ with probabilities $p, q_{1}$, and $q_{2}$, respectively. Then

$$
\frac{\partial^{2} \sigma}{\partial \mathrm{q}_{1} \partial \mathrm{q}_{2}}=\sum_{\substack{k, m=0 \\ k+m \leqq N-2}}^{N-2} v_{k m} p^{N-k-m-2} q_{1}^{k} q_{2}^{m} .
$$

When the number of bonds in $G$ goes to infinity, the appropriate limits of $v_{k m}$ yield exact formulas for the second mixed partial of $\sigma$. When $G$ is a hypercubic sample of the lattice with side $L$, then $N \sim d L^{d}$. In this case we consider $\sigma^{*}\left(q_{1}, q_{2}\right)$ as in (2.3), and assume for $\varepsilon=0$ that the infinite volume limits of the derivatives exist except at the critical probabilities (existence of these derivatives for $\varepsilon_{1}, \varepsilon_{2}>0$ will be proven subsequently). Then analogous to Lemma 3.2, with an analogous proof, we have

Lemma 3.4. For the lattice in $d \geqq 2$ with bond conductivities $1, \varepsilon_{1} \geqq 0$, and $\varepsilon_{2} \geqq 0$ with probabilities $p, q_{1}$, and $q_{2}$, respectively,

$$
p^{2} \frac{\partial^{2} \sigma^{*}}{\partial q_{1} \partial q_{2}}=\lim _{k, m, L \rightarrow \infty} L^{2-d} v_{k m} /\left(\begin{array}{c}
N \\
k
\end{array}\right)\left(\begin{array}{c}
N-k \\
m
\end{array}\right)
$$


where the simultaneous limits of $k, m$ and $L \rightarrow \infty$ are taken so that

$$
\begin{aligned}
& \lim _{k, L \rightarrow \infty} \frac{k}{N}=\lim _{k, L \rightarrow \infty} \frac{k}{d L^{d}}=q_{1}, \\
& \lim _{m, L \rightarrow \infty} \frac{m}{N}=\lim _{m, L \rightarrow \infty} \frac{m}{d L^{d}}=q_{2} .
\end{aligned}
$$

In order to derive a perturbation expansion of $\frac{\partial^{2} \sigma^{*}}{\partial q_{1} \partial q_{2}}$ around a homogeneous medium $\left(\varepsilon_{1}=\varepsilon_{2}=1\right)$, we first need a perturbation expansion of $\sigma^{*}$ analogous to (3.20), which we shall obtain from the analogue of (2.13) [42]. First, the threecomponent version of $m(\varepsilon)$ in (2.11) is

$$
m\left(\varepsilon_{1}, \varepsilon_{2}\right)=\sigma^{*}=\int_{\Omega} P(d \omega)\left(\varepsilon_{1} \chi_{1}+\varepsilon_{2} \chi_{2}+\chi_{3}\right) E_{k}^{k} .
$$

Again this maps the product of upper half-planes to the upper half-plane. Now define

$$
F\left(s_{1}, s_{2}\right)=1-m\left(\varepsilon_{1}, \varepsilon_{2}\right), \quad s_{1}=1 /\left(1-\varepsilon_{1}\right), \quad s_{2}=1 /\left(1-\varepsilon_{2}\right) .
$$

Then the analogue of (2.13) is

$$
F\left(s_{1}, s_{2}\right)=\int_{\Omega} P(d \omega)\left(\frac{1}{s_{1}} \chi_{1}+\frac{1}{s_{2}} \chi_{2}\right)\left[\left(I+\frac{1}{s_{1}} \Gamma \chi_{1}+\frac{1}{s_{2}} \Gamma \chi_{2}\right)^{-1} e_{k}\right] \cdot e_{k} .
$$

For $\left|s_{1}\right|>1$ and $\left|s_{2}\right|>1$, (3.34) can be expanded about a homogeneous medium $\left(\varepsilon_{1}=\varepsilon_{2}=1\right.$ or $\left.s_{1}=s_{2}=\infty\right)$ to obtain

$$
\begin{aligned}
F\left(s_{1}, s_{2}\right)= & \int_{\Omega} P(d \omega)\left[\left(\frac{\chi_{1}}{s_{1}}+\frac{\chi_{2}}{s_{2}}-\frac{\chi_{1} \Gamma \chi_{1}}{s_{1}^{2}}-\frac{\chi_{2} \Gamma \chi_{2}}{s_{2}^{2}}\right.\right. \\
& \left.\left.-\frac{\left(\chi_{1} \Gamma \chi_{2}+\chi_{2} \Gamma \chi_{1}\right)}{s_{1} s_{2}}+\ldots\right) e_{k}\right] \cdot e_{k} .
\end{aligned}
$$

By calculations similar to those mentioned in Sect. (3.1), the coefficients in (3.35) can be calculated to second order,

$$
1-\sigma^{*}=\frac{q_{1}}{s_{1}}+\frac{q_{2}}{s_{2}}+\frac{\left(q_{1}-q_{1}^{2}\right) / d}{s_{1}^{2}}+\frac{\left(q_{2}-q_{2}^{2}\right) / d}{s_{2}^{2}}-\frac{2 q_{1} q_{2} / d}{s_{1} s_{2}}+\ldots
$$

It is this expansion which will yield an expansion of $\frac{\partial^{2} \sigma^{*}}{\partial q_{1} \partial q_{2}}$, once we have established analyticity of $\sigma^{*}$ in $q_{1}$ and $q_{2}$, which is contained in

Theorem 3.4. Let $\sigma^{*}$ be the bulk conductivity of the bond lattice in any dimension with random bond conductivities $\varepsilon_{1}, \varepsilon_{2}$ and 1 with probabilities $q_{1}, q_{2}$ and $p=1-q_{1}-q_{2}$, respectively. Then for every $\varepsilon_{1}, \varepsilon_{2}>0$, there exists an open neighborhood $V_{\varepsilon_{1} \varepsilon_{2}} \supset[0,1]$ $\times[0,1]$ in $\mathbb{C}^{2}$ such that $\sigma^{*}\left(q_{1}, q_{2}\right)$ is analytic in $V_{\varepsilon_{1} \varepsilon_{2}}$.

Proof. The basic idea of the proof is the same as that of Theorem 3.2, i.e., to prove uniform convergence of (3.36). However, the coefficients in (3.36) are not the moments of a measure, as was the case for two-component media in (3.17), so that the bound $\mu_{n}(p) \leqq 1$, which was important in the proof, was immediate. Here we 
must work a little. Fix $s_{1}, s_{2}>1$ and assume $s_{1}<s_{2}$. Let

$$
A=\chi_{1}+\frac{s_{1}}{s_{2}} \chi_{2}
$$

so that (3.34) can be written as

$$
F\left(s_{1}, s_{2}\right)=\int_{\Omega} P(d \omega) \frac{1}{s_{1}} A\left[\left(I+\frac{1}{s_{1}} \Gamma A\right)^{-1} e_{k}\right] \cdot e_{k} .
$$

An easy calculation (using the orthogonality of $\chi_{1}$ and $\chi_{2}$ ) shows that

$$
\|A\| \leqq 1,
$$

where the norm in (3.39) is operator norm in $L^{2}(\Omega, P)$. Since $\Gamma$ is a projection operator, $\Gamma^{2}=\Gamma$, it also satisfies

$$
\|\Gamma\| \leqq 1 .
$$

Using $A$ and $\Gamma$ we can rewrite (3.36) as

or

$$
F\left(s_{1}, s_{2}\right)=\int_{\Omega} P(d \omega) e_{k} \cdot\left[\frac{A}{s_{1}}+\frac{A \Gamma A}{s_{1}^{2}}+\frac{A(\Gamma A)^{2}}{s_{1}^{3}}+\ldots\right] e_{k}
$$

$$
F\left(s_{1}, s_{2}\right)=\sum_{n=1}^{\infty} \frac{f_{n}\left(q_{1}, q_{2}\right)}{s_{1}^{n}},
$$

where the $f_{n}\left(q_{1}, q_{2}\right)$ are $n^{\text {th }}$ order polynomials in $q_{1}$ and $q_{2}$ which satisfy by CauchySchwartz, (3.39) and (3.40) the inequality

$$
\left|f_{n}\left(q_{1}, q_{2}\right)\right| \leqq 1, \quad q_{1}, q_{2} \in[0,1] .
$$

Now the argument parallels the proof of Theorem 3.2. Let

$$
W^{2}=\left\{q_{1} \in \mathbb{C} \mid q_{1} \notin[0,1]\right\} \times\left\{q_{2} \in \mathbb{C} \mid q_{2} \notin[0,1]\right\} .
$$

Conformally map $W^{2}$ onto the unit polydisk $D^{2}$ in $\left(z_{1}, z_{2}\right)$-space, so that $\left(q_{1}, q_{2}\right)$ $=(\infty, \infty)$ gets mapped to $\left(z_{1}, z_{2}\right)=(0,0)$, and $[0,1] \times[0,1]$ gets mapped to the distinguished boundary of $D^{2}$, i.e., to the torus $T^{2}=\left\{\left|z_{1}\right|=1\right\} \times\left\{\left|z_{2}\right|=1\right\}$. Since $f_{n}\left(q_{1}, q_{2}\right)$ is an $n^{\text {th }}$ order polynomial in $q_{1}$ and $q_{2}, f_{n}\left(z_{1}, z_{2}\right)$ has at worst an $n^{\text {th }}$ order pole (in each variable) at $\left(z_{1}, z_{2}\right)=(0,0)$. Thus $\left(z_{1} z_{2}\right)^{n} f_{n}\left(z_{1}, z_{2}\right)$ is analytic in $D^{2}$. Since $\left|f_{n}\left(z_{1}, z_{2}\right)\right| \leqq 1$ on $T^{2}$, it also satisfies $\left|f_{n}\left(z_{1}, z_{2}\right)\right| \leqq 1$ on all of $\partial D^{2}$, since if $\left|z_{1}\right|=1$ is fixed, $f\left(z_{1}, z_{2}\right)$ as a function of $z_{2} \in D$ attains its maximum on the boundary $\left|z_{2}\right|=1$, so that $\left|f\left(z_{1}, z_{2}\right)\right| \leqq 1$ when $\left|z_{1}\right|=1$ and $\left|z_{2}\right|<1$, and vice versa. Then by the maximum modulus principle for analytic functions of two variables applied to $\left(z_{1} z_{2}\right)^{n} f_{n}\left(z_{z}, z_{2}\right)$

$$
\left|f_{n}\left(z_{1}, z_{2}\right)\right| \leqq \frac{1}{\left|z_{1} z_{2}\right|^{n}}, \quad\left(z_{1}, z_{2}\right) \in D^{2} .
$$

Now, for any small $\delta^{\prime}>0$, there is a small $\delta>\delta^{\prime}>0$ such that in the product annulus $A_{\delta^{\prime}}=\left\{1 \geqq\left|z_{1}\right|>1-\delta^{\prime}\right\} \times\left\{1 \geqq\left|z_{2}\right|>1-\delta^{\prime}\right\}$,

$$
\left|f_{n}\left(z_{1}, z_{2}\right)\right| \leqq(1+\delta)^{n}, \quad\left(z_{1}, z_{2}\right) \in A_{\delta^{\prime}}
$$

For our given $s_{1}>1$ and $s_{2}>1$ (or $\varepsilon_{1}, \varepsilon_{2}>0$ ), we can choose $\delta$ and $\delta^{\prime}$ so that

$$
\left|f_{n}\left(q_{1}, q_{2}\right)\right| \leqq(1+\delta)^{n}, \quad\left(q_{1}, q_{2}\right) \in V_{\varepsilon_{1} \varepsilon_{2}},
$$


where $V_{\varepsilon_{1} \varepsilon_{2}}$ conformally maps onto $A_{\delta^{\prime}}$. Then (3.42) converges uniformly in $V_{\varepsilon_{1} \varepsilon_{2}}$, which proves the theorem.

Remark. The above theorem applies to $N$-component infinitely interchangeable media in the continuum, as well as the lattice.

Now that we have established the analyticity, we can differentiate (3.36) to obtain

Theorem 3.5. Let $\sigma^{*}$ be the bulk conductivity of the bond lattice in any $d \geqq 1$ with random bond conductivities $\varepsilon_{1}, \varepsilon_{2}$ and 1 with probabilities $q_{1}, q_{2}$ and $p=1-q_{1}-q_{2}$, respectively. Then,

$$
\frac{\partial^{2} \sigma^{*}}{\partial q_{1} \partial q_{2}}=\frac{2 / d}{s_{1} s_{2}}+O\left(\frac{1}{s_{1} s_{2}^{2}}\right)+O\left(\frac{1}{s_{1}^{2} s_{2}}\right) .
$$

\section{Regimes of Convexity}

Since our principal focus of this work is the behavior of $\frac{d^{2} \sigma^{*}}{d p^{2}}$, it is useful to outline the regimes in $\varepsilon$ and $p$ for which $\sigma^{*}(p)$ appears to be convex. We shall first discuss numerical results, and then analytic results addressing this issue.

\subsection{Numerical Results}

Let $G$ be a graph having bond conductivities 1 and $\varepsilon \geqq 0$ with probabilities $p$ and $q=1-p$, and let $\sigma(p)$ be its effective conductivity function. We have computed $\frac{d^{2} \sigma}{d p^{2}}$ for a variety of graphs, including the square lattice, the triangular lattice, the hexagonal lattice, trees (Bethe lattice), ladders, the Wheatstone bridge, and others. In particular, we have computed the $\gamma_{k}$ in (3.4), or approximations of them, for these various lattices.

To summarize our results, away from $p=1$ networks we have considered have $\sigma(p)$ convex for any $\varepsilon \geqq 0$, and for both bond and site problems. (In site problems, when a "site" is assigned conductivity $\varepsilon$, this means that all attached bonds are assigned conductivity $\varepsilon$.) Near $p=1$, however, bond problems typically are not convex when $\varepsilon$ is small enough, while site problems typically are convex for all $\varepsilon \geqq 0$. For a typical bond problem, if we start with $\varepsilon$ close to 1 and allow it to shrink, $\left.\frac{d^{2} \sigma}{d p^{2}}\right|_{p=1}$ is positive until, say $\varepsilon \approx 0.1$, below which it becomes and stays slightly negative all the way down to $\varepsilon=0$. This concavity near $p=1$ for $\varepsilon=0$ is consistent with the results in [19] for the square lattice. Furthermore, the convexity near $p=1$ for site problems is consistent with the results of [20] for the square lattice. We see then for the site problem, $\sigma^{*}(p)$ for the square lattice with $\varepsilon=0$ appears to be convex for all $p$.

In the specific case of the bond problem for the $d=2$ lattice with $\varepsilon=0$, as $p$ increases from $p_{c}=\frac{1}{2}$ to 1 , what apparently happens is that $\sigma^{*}(p)$ starts off convex at $p=\frac{1}{2}$, but eventually the curve "turns over," i.e., become slightly concave, and stays this way until $p=1$. We note that this effect is subtle, because away from the critical regime $\left(p \approx \frac{1}{2}\right)$, the graph of $\sigma^{*}(p)$ looks nearly linear, where effective medium theory 
[6] is believed to provide a very good approximation. In fact, an expression for $\left.\frac{d^{2} \sigma^{*}}{d p^{2}}\right|_{p=1}$ is found in [19] and is numerically evaluated, with a result of about -0.21 , which supports the accuracy of effective medium theory near $p=1$. This result agrees with our calculations of $\frac{d^{2} \sigma^{*}}{d p^{2}}$, which give a value of about -0.2 at $p=1$ for a $15 \times 15$ sample of the square lattice. The calculation of $\left.\frac{d^{2} \sigma^{*}}{d p^{2}}\right|_{p=1}[19]$ is somewhat involved, and it is not at all obvious that the result will be negative, which gives motivation for the first of the analytic results.

\subsection{Analytic Results}

Casual inspection of numerical simulations and sketches in review articles of the graph of $\sigma^{*}(p)$ for the $d=2$ bond problem with $\varepsilon=0$ suggest that $\sigma^{*}(p)$ is a convex function of $p$. Furthermore, the expansion of $\frac{d^{2} \sigma^{*}}{d p^{2}}$ for $\varepsilon>0$ in (3.24) in powers of $(1-\varepsilon)$ has positive coefficients to $4^{\text {th }}$ order in $(1-\varepsilon)$, again suggesting convexity. However, the following result establishes the contrary.

Theorem 4.1 ( $d=2$ bond problem). If $\varepsilon=0, \sigma^{*}(p)$ cannot be convex for all $p \in[0,1]$ with strict convexity in some open interval.

Proof. It is known $[10,19]$ that with $\varepsilon=0$,

$$
\left.\frac{d \sigma^{*}}{d p}\right|_{p=1}=2 .
$$

The existence of this derivative has been proven in [14] for a class of continuum problems analogous to our bond problem, and presumably the arguments hold in the present situation. Now, a straight line with slope 2 passing through $\sigma^{*}(1)=1$ intersects the $p$-axis at $p=\frac{1}{2}$. If $\sigma^{*}(p)$ is convex for all $p$, and strictly convex in some open interval, its graph lies above this straight line, and it intersects the $p$-axis somewhere less than $p=\frac{1}{2}$, so that $p_{c}<\frac{1}{2}$, which contradicts the rigorous result [12] that $p_{c}=\frac{1}{2}$.

It was mentioned in Sect. 4.1 that $\sigma_{s}^{*}(p)$ (we have added an "s" subscript to avoid confusion) for the $d=2$ site problem with $\varepsilon=0$ is numerically found to be convex for all $p \in[0,1]$. Under this assumption, using an idea similar to the proof of Theorem 4.1, with the 2 in (4.1) replaced by $\pi[17,20]$, we can easily show that the critical probability $\hat{p}_{c}$ for the site problem in $d=2$ satisfies $\hat{p}_{c} \leqq 1-1 / \pi$, where $\hat{p}_{c}$ is believed to be about 0.59 , whereas $1-1 / \pi \approx 0.68$.

In the previous section on numerical results it was found that convexity of $\sigma^{*}(p, \varepsilon)$ for the bond problem appears to be lost only when $\varepsilon$ becomes small enough. The following result, which follows immediately from Theorem 3.3, provides some basis for this observation.

Theorem 4.2 ( $d \geqq 1$ bond problem). For $\varepsilon$ sufficiently close to $1, \sigma^{*}(p)$ is convex for all $p \in[0,1]$.

Remark. Theorem 4.2 holds for infinitely interchangeable media in the continuum as well. 
Finally we come to the principal result of this section, which was proven in [21].

Theorem 4.3 ( $d=2$ bond problem). For every $\varepsilon>0$, there exists an open neighborhood $\mathscr{U}_{\varepsilon} \subset[0,1]$ containing $p_{c}=\frac{1}{2}$ such that $\sigma^{*}(p)$ is convex in $\mathscr{U}_{\varepsilon}$.

The idea of the proof is to calculate the second derivative of $\sigma^{*}(p)$ at $p=1 / 2$, and show that it is positive by relating $\sigma^{*}(1 / 2-h)$ to $\sigma^{*}(1 / 2+h)$ for small $h$, via the duality relation $\sigma^{*}(p) \sigma^{*}(1-p)=\varepsilon$ [43]. This duality relation is a consequence of the Keller-Dykhne Interchange Theorem $[44,45]$, which holds for general stationary random media.

Due to the broad generality of both the Interchange Theorem and the analyticity in Theorem 3.2, the above convexity result holds for the large class of infinitely interchangeable media in the continuum as well. For example, a hexagonal chessboard structure with conductivities 1 and $\varepsilon \geqq 0$ randomly assigned to the hexagons has $p_{c}=\frac{1}{2}[14,46]$ (when $\varepsilon=0$ ) and Theorem 4.3 applies directly as stated. Its importance is that it indicates for actual systems, where the insulating material has a very small, but non-zero conductivity, $\sigma^{*}(p)$ has a convex shape in the transition regime around $p_{c}$, so that the critical exponent $t$ measured from data very near $p_{c}$ will satisfy $t \geqq 1$.

\section{Convexity and Exponent Inequalities for the Hierarchical NLB Model}

We are now ready to compute the second derivative of the bulk conductivity for the NLB model. Before we introduce the model, though, it is very instructive to describe how the geometry of a graph determines the convexity of its conductivity function $\sigma(p)$. More precisely, we shall investigate how pairs of bonds in series or in parallel contribute to determine the sign of the second derivative. It is this subtle interplay which will be quantitatively captured in the NLB calculation, and it is useful to first understand it in simple settings. Subsequently we shall define the NLB model and then compute the second derivative of the bulk conductivity for it.

\subsection{Geometry of Convexity}

Since we are now in the $\varepsilon=0$ case, it is useful to simplify the notation of (3.1) and (3.2). Thus, for any finite graph $B$ with bonds of unit conductivity [which will eventually be a backbone configuration $B(p)]$, let

$$
\begin{gathered}
\delta \sigma(B)=\sum_{b_{\imath} \in B}\left[\sigma_{i}(1)-\sigma_{i}(0)\right], \\
\delta^{2} \sigma(B)=\sum_{\substack{b_{\imath}, b_{j} \in B \\
b_{i} \neq b_{j}}}\left[\sigma_{i j}(1,1)+\sigma_{i j}(0,0)-\sigma_{i j}(1,0)-\sigma_{i j}(0,1)\right],
\end{gathered}
$$

where in (5.1) and (5.2), $\sigma_{i}(1)=\sigma_{i j}(1,1)=\sigma(B)$, the conductivity of $B$ measured between two vertices, $\sigma_{i}(0)$ is the conductivity of $B$ with bond $b_{i}$ removed, $\sigma_{i j}(0,0)$ is the conductivity of $B$ with bonds $b_{i}$ and $b_{j}$ removed, $\sigma_{i j}(1,0)$ is the conductivity of $B$ with bond $b_{j}$ removed, and vice versa for $\sigma_{i j}(0,1)$.

In order to illustrate how the geometry of a graph $B$ determines the sign of $\delta^{2} \sigma(B)$, let us consider the following very simple graph, which in some sense forms a fundamental building block for the NLB model. This graph is in the shape of a $Y$, where current flows in through the single leg and out through the two arms. For 
simplicity let each "limb" of the $Y$ be composed of one bond of unit conductivity, with $b_{1}$ the leg and $b_{2}$ and $b_{3}$ the arms. Consider now $\delta^{2} \sigma(Y)=2\left(\delta_{12}+\delta_{23}+\delta_{13}\right)$, where the $\delta_{i j}$ are the summands in (5.2). Elementary calculation shows that $\delta_{12}=\delta_{13}=+\frac{1}{6}$ and $\delta_{23}=\frac{-1}{3}$, so that $\delta^{2} \sigma(Y)=0$. The important point to note is that $(2,3)$ is a pair of bonds in parallel corresponding to $\delta_{23}<0$, while $(1,2)$ and $(1,3)$ are pairs in series corresponding to $\delta_{12}=\delta_{13}>0$.

For an arbitrary graph $G$, there is no particular reason why the positive $\delta_{i j}$ 's should outweigh the negative ones. However, for a graph $B$ that is sufficiently "stringy," positive contributions from series pairs in a given string should tip the balance to a net positive $\delta^{2} \sigma(B)>0$. For example, if we replace each bond in $Y$ above with two bonds in series, and call the new graph $S_{2} Y$, then $\delta^{2} \sigma\left(S_{2} Y\right)>0$. The reason is that the three series pairs in the leg and two arms give new, positive contributions to $\delta^{2} \sigma\left(S_{2} Y\right)$ which were not present in $\delta^{2} \sigma(Y)$. Such considerations led us to the following theorem. Two proofs were given in [21], the first which is essentially included in the NLB calculation and will be omitted here, and the second which will form the basis of our subsequent analysis of the third derivative, and will be given here.

Theorem 5.1. Let $G$ be any finite connected graph with unit bond conductivities, and let $S_{n} G$ be a new graph formed by replacing each bond of $G$ with $n$ bonds in series. Then

$$
\delta^{2} \sigma\left(S_{n} G\right)=n \delta^{2} \sigma(G)+(n-1) \delta \sigma(G), \quad \delta \sigma(G) \geqq 0 .
$$

Consequently, if $G$ satisfies

$$
C=\delta^{2} \sigma(G)+\delta \sigma(G)>0
$$

then

$$
\delta^{2} \sigma\left(S_{n} G\right) \sim C n, \quad C>0, n \rightarrow \infty .
$$

Proof. Let the conductivity function of $S_{n} G$ be denoted by $\hat{\sigma}(p)$, and that of $G$ be denoted by $\sigma(p)$. Then

$$
\hat{\sigma}(p)=\frac{1}{n} \sigma\left(p^{n}\right) .
$$

Differentiating both sides of (5.6) twice with respect to $p$ and setting $p=1$ yields

$$
\left.\frac{d^{2} \hat{\sigma}}{d p^{2}}\right|_{p=1}=\left.n \frac{d^{2} \sigma}{d p^{2}}\right|_{p=1}+\left.(n-1) \frac{d \sigma}{d p}\right|_{p=1},
$$

which is equivalent to (5.3).

This proof was a joint observation with S. Goldstein.

Remark. $S_{n} G$ is composed of a graph $G$ whose elements are strings of $n$ bonds in series. Removal of any one of these bonds converts all the other bonds in that string into dangling bonds. Their subsequent removal has no effect on the conductivity, and this effect is the source of the positive, second term in (5.3). We thus call $S_{n}$ a "convexity improving" transformation on graphs, as it typically transforms a graph with possibly negative $\delta^{2} \sigma(G)$ into one with positive $\delta^{2} \sigma\left(S_{n} G\right)$. 


\subsection{The Hierarchical Node-Link-Blob Model}

In order to define the NLB model, we must introduce the notion of correlation length for $p>p_{c}$. For the infinite bond lattice in $d \geqq 2$ with a fraction $p$ of occupied bonds let

$\tau^{f}(0, x)=\operatorname{Prob}_{p}\{0$ and $x$ belong to the same finite cluster of occupied bonds $\}$.

Then the correlation length can be defined by

$$
\frac{1}{\xi(p)}=\lim _{x \rightarrow \infty}-\frac{1}{|x|} \log \tau^{f}(0, x),
$$

where the limit is taken as $x$ moves out to infinity in a fixed direction. This limit was proven to exist for $p>p_{c}$ in [47]. We shall assume that $\xi$ diverges with an exponent $v$,

$$
\xi(p) \sim\left(p-p_{c}\right)^{-v}, \quad p \rightarrow p_{c}^{+} .
$$

We now describe the model. The NLB graph is a "super-lattice" consisting of nodes, spatially separated by a correlation length $\xi$, which are connected by $1^{\text {st }}$ order necklaces composed of strings (links) and $1^{\text {st }}$ order beads (blobs), as in Fig. 1 a. The beads themselves have a hierarchical structure, as shown in Fig. 1 b, consisting of two $2^{\text {nd }}$ order necklaces in parallel, and so on, in a self-similar fashion to order $N$, for an arbitrary, large integer $N$ ( $N$ no longer refers to the the number of bonds in a graph). We assume that any $k^{\text {th }}$ order necklace has $\beta$ beads on it, for an arbitrary, large integer $\beta$, and that each pair of beads is joined by a string of $n_{k}$ bonds, so that there are a total of $\beta n_{k}$ string bonds on each necklace. (Note that the figure is drawn with $\beta-1$ beads per necklace, but we take the number of beads to be $\beta$ for computational simplicity.) The $\beta n_{1}$ string bonds on any $1^{\text {st }}$ order necklace are called singly connected - because removal of one of them breaks the connection between nodes separated by $\xi$. All the rest of the bonds in the NLB graph are multiply connected, and among

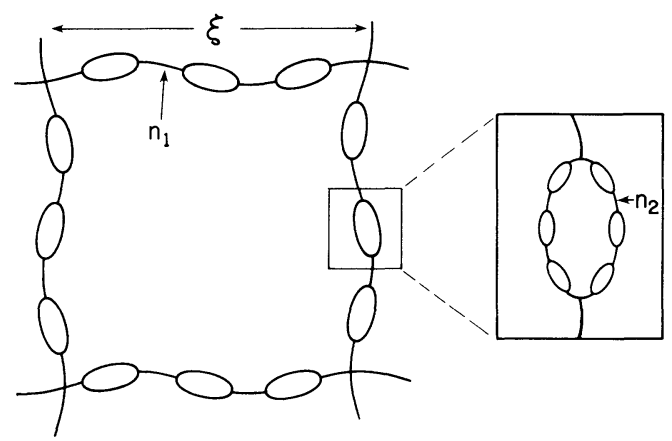

(a)

Fig. 1a, b. Node-link-blob model of the conducting backbone near $p_{c}$. In a, the nodes are a correlation length $\xi$ apart, and are connected by necklaces of beads (blobs) and strings (links) with $n_{1}$ bonds connecting two beads. The beads have a self-similar structure, as shown in $\mathbf{b}$, with $n_{2}$ bonds connecting two beads 
these it is useful to identify the $\beta n_{2}$ string bonds on a $2^{\text {nd }}$ order necklace as doubly connected, since it is possible to remove two of them (in parallel) and break a connection between nodes. There are then a total of $2 \beta^{2} n_{2}$ doubly connected beads between two nodes. Based on a result of Coniglio's [24] implying in our context that the number of singly and doubly connected bonds between the nodes both diverge with exponent 1 as $p \rightarrow p_{c}^{+}$, we assume that $n_{1}=2 \beta n_{2}$. (That the number of singly connected bonds diverges like $\left(p-p_{c}\right)^{-1}$ was previously shown in [48].) Due to self-similarity, we assume that

$$
n_{j-1}=2 \beta n_{j}, \quad j=2, \ldots, N .
$$

Relation (5.11) can be used to solve for the $n_{j}, j>1$, in terms of $n_{1}$, with

$$
n_{2}=\frac{n_{1}}{2 \beta}, n_{3}=\frac{n_{1}}{4 \beta^{2}}, \ldots, n_{j+1}=\frac{n_{1}}{2^{j} \beta^{j}}, \ldots, \quad j=1, \ldots, N-1 .
$$

In this model the percolation limit $p \rightarrow p_{c}^{+}$is characterized by the limits $n_{1} \rightarrow \infty$, $\beta \rightarrow \infty, N \rightarrow \infty$ and $\xi \rightarrow \infty$, so that the lengths of all orders of necklaces, and consequently the sizes and numbers of all orders of blobs, diverge as $p \rightarrow p_{c}^{+}$. The basic calculations, though, will be done for fixed, large $n_{1}, \beta, N$, and $\xi$, and we denote the NLB graph by $A\left(n_{1}\right)$.

It is important to remark how to construct $A\left(n_{1}\right)$. Let $G$ be the hypercubic lattice in $d \geqq 2$. Then $A\left(n_{1}\right)$ is constructed by replacing each bond of $G$ by a $1^{\text {st }}$ order necklace with hierarchical structure to order $N$, and spatially separating the nodes of $G$ by a correlation length $\xi$. We also note that it is not important that we choose the hypercubic lattice as the base lattice $G$. For example, say in $d=2$, we could choose $G$ to be the hexagonal lattice, whereby each node of $A\left(n_{1}\right)$ would be a junction of three necklaces rather than four, which is more common in actual percolation clusters [D. Fisher, priv. comm.].

With the above construction in mind, and in view of Theorem 5.1, one can imagine replacing the bonds of the base lattice $G$ by $n$ bonds in series, rather than a necklace with hierarchical structure, and again separating the nodes by a correlation length $\xi$. This simpler construction is essentially the node-link model of Skal and Shlovskii [48] and de Gennes [49]. However, due to the presence of only singly connected bonds, this model was found to be inadequate, particularly in $d=2,3$. In other words, an accurate description of the backbone structure near $p_{c}$ must include the blobs, which contain the multiply connected bonds, and give the model loops on many length scales smaller that the correlation length. This is precisely what $A\left(n_{1}\right)$ incorporates.

From the point of view of Sect. 5.1, it is not at all surprising that the node-link model would yield a positive second derivative, as evidenced by Theorem 5.1, where the node-link model is essentially $S_{n} G$ for a reasonable base lattice $G$. This is because each bond of $G$ is replaced by $n$ bonds in series, which as we have noted improves the convexity. However, in the NLB model, we are replacing each bond of $G$ with just as many bonds in parallel as in series, at each level of the hierarchy, i.e., $n_{1}=2 \beta n_{2}, n_{2}=2 \beta n_{3}$, and so on. Our subsequent calculations will show, nevertheless, that convexity still wins.

\subsection{Calculation of the Second Derivative for the NLB Graph}

We now proceed to the calculation of the asymptotics of $\delta^{2} \sigma^{*}\left(A\left(n_{1}\right)\right)$ as $n_{1}, \beta, N$, and $\xi \rightarrow \infty$, for a fixed base lattice $G$, the hypercubic lattice in $d \geqq 2$. Our first step is 
to relate the bulk quantity $\delta^{2} \sigma^{*}\left(A\left(n_{1}\right)\right)$ to the unscaled $\delta^{2} \sigma\left(A\left(n_{1}\right)\right)$ in (5.2) with $B=A\left(n_{1}\right)$. Since $\delta^{2} \sigma$ is linear in $\sigma$, it suffices to obtain the scaling for the conductivity itself. Let $G_{L}$ be a hypercubic sample of $G$ with side $L$, and let $z_{0}$ be the conductivity of a necklace. Furthermore, let $A_{L}$ be $G_{L}$ with each bond replaced by a necklace, and again, its nodes separated by $\xi$. Then one side of $A_{L}$ is clearly $\xi L$, so that

$$
\sigma^{*}\left(A_{L}\right)=\frac{\sigma\left(A_{L}\right)}{(L \xi)^{d-2}}=\frac{z_{0} \sigma\left(G_{L}\right)}{(L \xi)^{d-2}} .
$$

Taking the $L \rightarrow \infty$ limit of (5.13) yields $\sigma^{*}(A)=z_{0} / \xi^{d-2}$, so that

$$
\delta^{2} \sigma^{*}\left(A\left(n_{1}\right)\right)=\frac{\delta^{2} \sigma\left(A\left(n_{1}\right)\right)}{\xi^{d-2}}, \quad \delta \sigma^{*}\left(A\left(n_{1}\right)\right)=\frac{\delta \sigma\left(A\left(n_{1}\right)\right)}{\xi^{d-2}} .
$$

In order to calculate $\delta^{2} \sigma\left(A\left(n_{1}\right)\right)$ from (5.2), we shall need the conductivities of $A$ with all possible pairings of bonds removed. To help us compute this, we shall need the conductivity of a single necklace with each type of bond removed, and also with each type of pair removed. To be clear, we refer to segments of string bonds on a $k^{\text {th }}$ order necklace as a $k^{\text {th }}$ order string. For example, the $1^{\text {st }}$ order strings comprise the set of all singly connected bonds, and the $2^{\text {nd }}$ order strings comprise the set of all doubly connected bonds, and so on.

We first compute the conductivities of a necklace with one bond removed. Let $z_{0}$ be the conductivity of a necklace with no bonds removed, $z_{1}$ be the conductivity of a necklace with one bond removed from a $1^{\text {st }}$ order string, and in general, $z_{k}$ be the conductivity of a necklace with one bond removed from a $k^{\text {th }}$ order string. Since a necklace is composed only of bonds in series and parallel, the $z_{k}$ are not difficult to compute. First we have

$$
\left(z_{0}\right)^{-1}=\beta n_{1}+\frac{\beta^{2} n_{2}}{2}+\frac{\beta^{3} n_{3}}{4}+\ldots+\frac{\beta^{N} n_{N}}{2^{N}},
$$

which becomes via (5.12),

$$
z_{0}=\frac{\alpha_{N}}{\beta n_{1}}
$$

where

Clearly $z_{1}=0$.

$$
\left(\alpha_{N}\right)^{-1}=\sum_{j=0}^{N}\left(\frac{1}{4}\right)^{j}, \quad \alpha_{N}<1
$$

Next we have

$$
\left(z_{2}\right)^{-1}=\beta n_{1}+(\beta-1)\left(\frac{\beta n_{2}}{2}+\frac{\beta^{2} n_{3}}{4}+\ldots\right)+\beta n_{2}+\frac{\beta^{2} n_{3}}{2}+\ldots,
$$

where in (5.18) and subsequently we shall usually omit writing the $N^{\text {th }}$ term for simplicity, it being understood that these are only finite sums.

Using (5.12), after some manipulation (5.18) becomes

$$
z_{2}=\frac{z_{0}}{1+\frac{\alpha_{N}\left(1 / \alpha_{N}-1\right)}{\beta}}
$$


In general, we have

Lemma 5.1. The conductivity $z_{k}$ of a necklace with one bond from a $k^{\text {th }}$ order string removed is

$$
z_{k}=\frac{z_{0}}{1+\frac{\gamma_{k}}{\beta^{k-1}}}, \quad k \geqq 2,
$$

where $z_{0}$ is given in (5.16) and (5.17), and $\gamma_{k}$ satisfies

$$
\gamma_{k}=\alpha_{N}\left(1 / \alpha_{N}-\sum_{j=0}^{k-2}\left(\frac{1}{4}\right)^{j}\right) /\left(2^{k-1}-1\right), \quad 0<\gamma_{k}<1, k \geqq 2 .
$$

For large $\beta,(5.20)$ can be expanded as

$$
z_{k}=z_{0}\left(1-\frac{\gamma_{k}}{\beta^{k-1}}+\frac{\gamma_{k}^{2}}{\beta^{2(k-1)}}-\ldots\right), \quad k \geqq 2 .
$$

Remark. The important point to note in (5.22) is that as $k$ increases, the deviation of $z_{k}$ from $z_{0}$ decreases geometrically fast in powers of $\beta$ :

We now must compute the conductivities of a necklace with all possible pairs of bonds removed from this necklace, which is somewhat more complicated than above. Our calculations will be divided into two primary groups. The first deals with pairs for which both bonds are members of a $k^{\text {th }}$ order string, the second deals with pairs for which one bond is in a $k^{\text {th }}$ order string while the other bond is in a $j^{\text {th }}$ order string, with $j \neq k$. Each of these two groups will contain two types of calculations. The first deals with pairs in series and the second with pairs in parallel.

First we compute $z_{k k}^{s}$, the conductivity of a necklace with two bonds in different $k^{\text {th }}$ order strings removed in series. We assume that the two bonds are members of different blobs of order $k-1$, so that the second removal is not redundant. It is most instructive to start with $k=1$ and work our way up to see the general pattern. For $k=1$, redundancy of the second removal is unavoidable, with $z_{11}^{s}=0$. For $k=2$, we have

$$
\begin{aligned}
\left(z_{22}^{s}\right)^{-1} & =\beta n_{1}+(\beta-2)\left(\frac{\beta n_{2}}{2}+\frac{\beta^{2} n_{3}}{4}+\ldots\right)+2\left(\beta n_{2}+\frac{\beta^{2} n_{3}}{2}+\ldots\right) \\
& =\frac{\beta n_{1}}{\alpha_{N}}+\frac{n_{1}}{2}\left(1+\frac{1}{4}+\frac{1}{16}+\ldots\right) .
\end{aligned}
$$

Then, in view of (5.16) and (5.17),

$$
z_{22}^{s}=\frac{z_{0}}{1+\frac{\alpha_{N} / \alpha_{N-1}}{2 \beta}} .
$$

In general, we have

$$
z_{k k}^{s}=\frac{z_{0}}{1+\frac{\gamma_{k k}^{s}}{\beta^{k-1}}}, \quad k \geqq 3
$$


where

$$
\gamma_{k k}^{s}=\alpha_{N}\left(1 / \alpha_{N-1}-\sum_{j=0}^{k-3}\left(\frac{1}{4}\right)^{j}\right) /\left(2^{k}-2\right), \quad k \geqq 3 .
$$

In the special case where the removal of the second bond is redundant, we call the conductivity $z_{k k}^{r}$, with $z_{k k}^{r}=z_{k}, k \geqq 2$.

Now we compute $z_{k k}^{p}$, the conductivity of a necklace with two bonds removed in parallel from different $k^{\text {th }}$ order strings. We shall first assume that the second removal does not break a necklace of order $k-1$. For $k=2$ this breakage is unavoidable, with $z_{22}^{p}=0$. For $k=3$ we have

$$
\begin{aligned}
\left(z_{33}^{p}\right)^{-1}= & \beta n_{1}+\frac{\beta^{2} n_{2}}{2}+(\beta-1)\left(\frac{\beta^{2} n_{3}}{4}+\frac{\beta^{3} n_{4}}{8}+\ldots\right) \\
& +(\beta-1)\left(\frac{\beta n_{3}}{4}+\frac{\beta^{2} n_{4}}{8}+\ldots\right)+\frac{\beta n_{3}}{2}+\frac{\beta^{2} n_{4}}{4}+\ldots \\
= & \frac{\beta n_{1}}{\alpha_{N}}+\frac{n_{1}}{4 \beta}\left(\frac{1}{16}+\frac{1}{64}+\ldots\right)
\end{aligned}
$$

so that

$$
z_{33}^{p}=\frac{z_{0}}{1+\frac{\alpha_{N}\left(1 / \alpha_{N-1}-1-1 / 4\right)}{\beta^{2}}} .
$$

In general, we have

$$
z_{k k}^{p}=\frac{z_{0}}{1+\frac{\gamma_{k k}^{p}}{\beta^{k-1}}}, \quad k \geqq 3
$$

where

$$
\gamma_{k k}^{p}=\alpha_{N}\left(1 / \alpha_{N-1}-\sum_{j=0}^{k-2}\left(\frac{1}{4}\right)^{j}\right) /\left(2^{k-2}-1\right), \quad k \geqq 3
$$

In the special case where the second removal breaks a necklace of order $k-1$, we call the resulting conductivity $z_{k k}^{b}$, and we clearly have $z_{k k}^{b}=z_{k-1}, k \geqq 2$. We summarize our results in

Lemma 5.2. The conductivity $z_{k k}^{s}\left(z_{k k}^{p}\right)$ of a necklace with two bonds in series (parallel) removed from different strings of order $k$, assuming that the second removal is not redundant (that the second removal does not break a necklace of order $k-1$ ) is

$$
z_{k k}^{s}=\frac{z_{0}}{1+\frac{\gamma_{k k}^{s}}{\beta^{k-1}}}, \quad k \geqq 2,
$$

where $\gamma_{k k}^{s}$ is given by (5.26). The same formula holds for $z_{k k}^{p}$, with $\gamma_{k k}^{p}$ in (5.30) replacing $\gamma_{k k}^{s}, k \geqq 3$. In the redundant (series) and breakage (parallel) cases excluded above,

$$
z_{k k}^{r}=z_{k}, \quad z_{k k}^{b}=z_{k-1}, \quad k \geqq 2 .
$$


For large $\beta,(5.31)$ can be expanded as

$$
z_{k k}^{s}=z_{0}\left(1-\frac{\gamma_{k k}^{s}}{\beta^{k-1}}+\left(\frac{\gamma_{k k}^{s}}{\beta^{k-1}}\right)^{2}-\ldots\right),
$$

and similarly for $z_{k k}^{p}, z_{k k}^{r}$, and $z_{k k}^{b}$.

Remark. Again the important point to note is that as $k$ increases, the deviation of $z_{k k}^{s}$ (and the others) from $z_{0}$ decreases geometrically fast in powers of $\beta$.

We now deal with the second class of pairs, where one bond is removed from a $j^{\text {th }}$ order string and the other bond is removed from a $k^{\text {th }}$ order string, $j \neq k$. Again it is most instructive to calculate a few simpler cases, which show the general pattern. First we consider $z_{j k}^{s}, j<k$, again under the assumption that the second removal is not redundant. With $j=1$, the redundancy is again unavoidable, with $z_{1 k}^{s}=0$. We now compute

$$
\begin{aligned}
\left(z_{23}^{s}\right)^{-1}= & \beta n_{1}+(\beta-2)\left(\frac{\beta n_{2}}{2}+\frac{\beta^{2} n_{3}}{4}+\ldots\right)+\frac{\beta n_{2}}{2} \\
& +(\beta-1)\left(\frac{\beta n_{3}}{4}+\frac{\beta^{2} n_{4}}{8}+\ldots\right) \\
& +\frac{\beta n_{3}}{3}+\frac{\beta^{2} n_{4}}{6}+\ldots+\beta n_{2}+\frac{\beta^{2} n_{3}}{2}+\ldots
\end{aligned}
$$

The point of the rearrangement now is to identify the terms associated with the removal of a single bond from a $2^{\text {nd }}$ order string,

$$
\begin{aligned}
\left(z_{23}^{s}\right)^{-1} & =\frac{\beta n_{1}}{\alpha_{N}}+\left(\frac{\beta n_{2}}{2}+\frac{\beta^{2} n_{3}}{4}+\ldots\right)+\frac{\beta n_{3}}{12}+\frac{\beta^{2} n_{4}}{24}+\ldots \\
& =\left(z_{2}\right)^{-1}+\frac{n_{1}}{3 \beta}\left(\frac{1}{16}+\frac{1}{64}+\ldots\right) .
\end{aligned}
$$

Then we can obtain

$$
z_{23}^{s}=\frac{z_{0}}{1+\frac{\gamma_{2}}{\beta}+\frac{\gamma_{3}}{\beta^{2}}} .
$$

Similar manipulations give in general

$$
z_{j k}^{s}=\frac{z_{0}}{1+\frac{\gamma_{j}}{\beta^{j-1}}+\frac{\gamma_{k}}{\beta^{k-1}}}, \quad k>j \geqq 2 .
$$

In the case where the second removal is redundant, $z_{j k}^{r}=z_{j}, k>j \geqq 1$.

Now we deal with the situation where the two bonds are removed in parallel, which will be denoted by $z_{j k}^{p}, k>j$. Note that in this case, with $k>j$, it is not possible to break a necklace of lower order. We first compute

$$
\begin{aligned}
\left(z_{23}^{p}\right)^{-1}= & \beta n_{1}+(\beta-1)\left(\frac{\beta n_{2}}{2}+\frac{\beta^{2} n_{3}}{4}+\ldots\right) \\
& +\beta n_{2}+(\beta-1)\left(\frac{\beta n_{3}}{2}+\frac{\beta^{2} n_{4}}{4}+\ldots\right) \\
& +\beta n_{3}+\frac{\beta^{2} n_{4}}{2}+\ldots
\end{aligned}
$$


Then we obtain after a little more work

$$
z_{23}^{p}=\frac{z_{0}}{1+\frac{\gamma_{2}}{\beta}+\frac{\gamma_{2} / 2}{\beta^{2}}}
$$

In general, similar calculations give

$$
z_{j k}^{p}=\frac{z_{0}}{1+\frac{\gamma_{j}}{\beta^{j-1}}+\frac{\gamma_{k-1} / 2}{\beta^{k-1}}}, \quad k>j \geqq 2 .
$$

We summarize our results in

Lemma 5.3. The conductivity $z_{j k}^{s}\left(z_{j k}^{p}\right)$ of a necklace with one bond removed from a $j^{\text {th }}$ order necklace and another bond in series (parallel) removed from a $k^{\text {th }}$ order necklace, $k>j$, assuming that the second removal is not redundant, is

$$
z_{j k}^{s}=\frac{z_{0}}{1+\frac{\gamma_{j}}{\beta^{j-1}}+\frac{\gamma_{k}}{\beta^{k-1}}}, \quad k>j \geqq 2,
$$

and

$$
z_{j k}^{p}=\frac{z_{0}}{1+\frac{\gamma_{j}}{\beta^{j-1}}+\frac{\gamma_{k-1} / 2}{\beta^{k-1}}}, \quad k>j \geqq 2 .
$$

In the series case, when the second removal is redundant,

$$
z_{j k}^{r}=z_{j}, \quad k>j \geqq 1 .
$$

For large $\beta,(5.41)$ and (5.42) can be expanded to yield

$$
\begin{gathered}
z_{j k}^{s}=z_{0}\left(1-\frac{\gamma_{j}}{\beta^{j-1}}-\frac{\gamma_{k}}{\beta^{k-1}}+O\left(\frac{1}{\beta^{2(j-1)}}\right)\right), \\
z_{j k}^{p}=z_{0}\left(1-\frac{\gamma_{j}}{\beta^{j-1}}-\frac{\gamma_{k-1} / 2}{\beta^{k-1}}+O\left(\frac{1}{\beta^{2(j-1)}}\right)\right) .
\end{gathered}
$$

Remark. The important point to note is that in (5.44) and (5.45), $z_{j k}^{s}$ and $z_{j k}^{p}$ match the expression for $z_{j}$ to order $1 / \beta^{j-1}$.

In order to state our principal theorem on the asymptotics of $\delta^{2} \sigma^{*}\left(A\left(n_{1}\right)\right)$, we must discuss the numerically verified condition under which the theorem is proved. The condition is as follows.

Hypothesis 5.1. Let $G$ be a lattice in d-dimensions (not necessarily hypercubic) with bond conductivities 1 and 0 in the volume fractions $p$ and $1-p$, and bulk conductivity function $\sigma^{*}(p)$. Then at least two derivatives of $\sigma^{*}(p)$ exist at $p=1$ and obey

$$
\kappa(G)=\delta \sigma^{*}(G)+\delta^{2} \sigma^{*}(G)=\left.\frac{d \sigma^{*}}{d p}\right|_{p=1}+\left.\frac{d^{2} \sigma^{*}}{d p^{2}}\right|_{p=1}>0 .
$$


For the square lattice in $d=2$, from (4.1) and the numerical results of Sect. 4, we see that (5.46) is satisfied. In higher dimensions [10]

$$
\delta \sigma^{*}(G)=\left.\frac{d \sigma^{*}}{d p}\right|_{p=1}=\frac{d}{d-1},
$$

while numerical simulation of $\sigma^{*}(p)$ in $d=3[6]$ and analytical solution of $\sigma^{*}(p)$ for the Bethe lattice, believed to represent large $d[50,51]$, are practically linear near $p=1$, so that $\left.\frac{d^{2} \sigma^{*}}{d p^{2}}\right|_{p=1}$ is small, as in $d=2$. Thus (5.74) is satisfied in higher $d p^{2}=1$
dimensions as well. Given (5.47), condition (5.46) amounts to saying that if $\frac{d^{2} \sigma^{*}}{d p^{2}}$ is negative, it must be small, i.e., the graph of $\sigma^{*}(p)$ must be nearly linear near $p=1$. However, the effective medium solution [10] to the bulk conductivity problem is a straight line with slope $d /(d-1)$. Hypothesis 5.1 just amounts to a particular consequence of the long accepted view that effective medium theory provides an accurate description of $\sigma^{*}(p)$ for $p$ near $p=1$. This is also true for more general lattices, such as periodic ones, like the hexagonal or triangular lattices, or presumably even quasiperiodic or random lattices. Thus Hypothesis 5.1 presumably holds in great generality, assuming the existence of the derivatives at $p=1$.

We now state

Theorem 5.2. Let $A\left(n_{1}\right)$ be the NLB graph formed from the hypercubic base lattice $G$, which is assumed to satisfy Hypothesis 5.1. We further assume the existence of the second mixed partial of $\sigma^{*}$ for three-component lattices at $p=1$, when one of the conductivities is 0 , as in Sect. 3. Then

$$
\delta^{2} \sigma\left(A\left(n_{1}\right)\right)=\alpha_{N} \kappa(G) \beta n_{1}+\sum_{j=0}^{\infty} \frac{c_{1}(j) n_{1}+c_{2}(j)}{\beta^{j}},
$$

where

$$
\left(\alpha_{N}\right)^{-1}=\sum_{j=0}^{\infty}\left(\frac{1}{4}\right)^{j} \quad \text { and } \quad \kappa(G)=\delta \sigma^{*}(G)+\delta^{2} \sigma^{*}(G)>0,
$$

and the $c_{1}(j)$ and $c_{2}(j)$ yield a convergent series in (5.48). Consequently, as $n_{1}, \beta, N$, and $\xi \rightarrow \infty$, we have

$$
\delta^{2} \sigma^{*}\left(A\left(n_{1}\right)\right) \sim \frac{\alpha_{N} \kappa(G) \beta n_{1}}{\xi^{d-2}}, \quad n_{1}, \beta, N, \xi \rightarrow \infty .
$$

with $\alpha_{N} \rightarrow 3 / 4$ as $N \rightarrow \infty$.

Proof. In order to calculate $\delta^{2} \sigma\left(A\left(n_{1}\right)\right)$, the conductivity of $A\left(n_{1}\right)$ with all possible pairings of bonds removed must be considered. Since $A\left(n_{1}\right)$ is formed by replacing the bonds of $G$ with hierarchical necklaces, the idea of the calculation is to compute appropriate sums of the conductivity of $G$ with the conductivities of one or two of its bonds changed to those conductivities calculated in the lemmas earlier in this section. By the results of Sect. 3, these sums can be handled by the perturbation of various derivatives of $\sigma^{*}$ for two- or three-component media at $p=1$.

In order to develop $\delta^{2} \sigma\left(A\left(n_{1}\right)\right)$ as the series in (5.48), we decompose its contributions as

$$
\delta^{2} \sigma\left(A\left(n_{1}\right)\right)=\sum_{\substack{j, k=1 \\ k \geqq j}}^{N} \delta_{j k},
$$


where $\delta_{j k}$ is the sum of all contributions to $\delta^{2} \sigma\left(A\left(n_{1}\right)\right)$ arising from pairs with one bond in a $j^{\text {th }}$ order string and the other bond in a $k^{\text {th }}$ order string, which is in either the same or a different $1^{\text {st }}$ order necklace. As in the proofs of the lemmas of this section, the structure of (5.48) will become apparent by considering the first few (dominant) terms of (5.51).

First we note that since all the conductivities in the lemmas have a factor of $z_{0}$ in them, and due to the homogeneity of effective conductivity, we can factor out this $z_{0}$ and consider $G$ with bonds of unit conductivity, and then when considering removal of appropriate bonds, instead of the $z_{k}$ and $z_{j k}$ in the lemma we shall replace the unit conductivity bonds of $G$ with bonds of conductivities

$$
\begin{gathered}
h_{k}=\frac{z_{k}}{z_{0}}, \quad h_{1}=0, \quad k \geqq 1, \\
h_{j k}=\frac{z_{j k}}{z_{0}}, \quad h_{1 k}=0, \quad j, k \geqq 1,
\end{gathered}
$$

for all appropriate forms of $h_{j k}$, namely parallel, series, and so on. We note further that even since we have factored out $\xi^{d-2}$ in dealing with $\delta^{2} \sigma\left(A\left(n_{1}\right)\right)$, the relevant conductivity will be the bulk conductivity $\sigma^{*}$ of $G$ (with appropriate bond conductivities changed), since in (5.13) when one removes the factor of $z_{0} / \xi^{d-2}$ one is still left with $\sigma\left(G_{L}\right) / L^{d-2}$, which converges to $\sigma^{*}(G)$ as $L \rightarrow \infty$. Henceforth in the proof $\sigma^{*}$ means the bulk conductivity scaled with respect to the base lattice $G$.

First we compute $\delta_{11}$. The calculation is essentially the first proof of Theorem 5.1 referred to earlier. We first deal with pairs in $\delta_{11}$ where both bonds are on the same necklace. Let $S_{1}^{k}$ denote the set of $\beta n_{1}$ string bonds on a first order necklace comprising the $k^{\text {th }}$ bond of the base lattice $G$. Since there are $\beta n_{1}\left(\beta n_{1}-1\right)$ of these types of pairs, we have

$$
\sum_{\substack{i, j \in S_{1}^{k} \\ i \neq j}}\left(\sigma_{i j}^{*}(1,1)+\sigma_{i j}^{*}(0,0)-\sigma_{i j}^{*}(1,0)-\sigma_{i j}^{*}(0,1)\right)=\beta n_{1}\left(\beta n_{1}-1\right)\left(\sigma_{k}^{*}(1)-\sigma_{k}^{*}(0)\right),
$$

where $\sigma_{k}^{*}(1)=\sigma^{*}(G)$ and $\sigma_{k}^{*}(0)$ is the conductivity of $G$ with the $k^{\text {th }}$ bond replaced by $h_{1}=0$, and for simplicity we have simplified the summation indices in the left from $b_{i}$ and $b_{j}$ to simply $i$ and $j$. Equation (5.54) follows since $\sigma_{i j}^{*}(0,0)=\sigma_{i j}^{*}(0,1)=\sigma_{i j}^{*}(1,0)$ in this case. Now we include also the pairs where both bonds are in different necklaces, so that

$$
\begin{aligned}
\delta_{11}= & z_{0}\left[\beta n_{1}\left(\beta n_{1}-1\right) \sum_{i \in G}\left(\sigma_{i}^{*}(1)-\sigma_{i}^{*}(0)\right)\right. \\
& \left.+\left(\beta n_{1}\right)^{2} \sum_{\substack{i, j \in G \\
i \neq j}}\left(\sigma_{i j}^{*}(1,1)+\sigma_{i j}^{*}(0,0)-\sigma_{i j}^{*}(1,0)-\sigma_{i j}^{*}(0,0)\right)\right] .
\end{aligned}
$$

Since $z_{0}=\alpha_{N} / \beta n_{1}$, carrying out the sums in (5.55) yields

$$
\delta_{11}=\alpha_{N} \beta n_{1}\left(\delta \sigma^{*}(G)+\delta^{2} \sigma^{*}(G)\right)-\alpha_{N} \delta \sigma^{*}(G),
$$

where the first term on the right in (5.56) corresponds to the first term on the right in (5.48). Note that the terms on the right in (5.56) involve the derivatives of $\sigma^{*}(p)$ only at $p=1$.

Now we consider $\delta_{12}$. Again we first look at pairs with the bonds in the same necklace. In addition to $S_{1}^{k}$ above, let $S_{2}^{k}$ denote the $2 \beta^{2} n_{2}=\beta n_{1}, 2^{\text {nd }}$ order string 
bonds in the necklace comprising the $k^{\text {th }}$ bond of $G$. We have

$$
2 \sum_{\substack{i \in S^{\mathrm{k}} \\ j \in S_{2}^{k}}}\left(\sigma_{i j}^{*}(1,1)+\sigma_{i j}^{*}(0,0)-\sigma_{i j}^{*}(1,0)-\sigma_{i j}^{*}(0,1)\right)=\left(\beta n_{1}\right)^{2}\left(\sigma_{k}^{*}(1)-\sigma_{k}^{*}\left(h_{2}\right)\right),
$$

since $\sigma_{i j}^{*}(0,0)=\sigma_{i j}^{*}(0,1)$ in this case. Including the pairs with bonds from different necklaces,

$$
\begin{aligned}
\delta_{12}= & z_{0}\left[\left(\beta n_{1}\right)^{2} \sum_{i \in G}\left(\sigma_{i}^{*}(1)-\sigma_{i}^{*}\left(h_{2}\right)\right)\right. \\
& \left.+\left(\beta n_{1}\right)^{2} \sum_{\substack{i, j \in G \\
i \neq j}}\left(\sigma_{i j}^{*}(1,1)+\sigma_{i j}^{*}\left(h_{1}, h_{2}\right)-\sigma_{i j}^{*}\left(1, h_{1}\right)-\sigma_{i j}^{*}\left(1, h_{2}\right)\right)\right] .
\end{aligned}
$$

Denote the first sum in (5.58) by $\sum_{1}$ and the second by $\sum_{2}$. By (3.1) and Lemma 3.2,

$$
\sum_{1}=\frac{d \sigma^{*}}{d p}\left(p=1, h_{2}\right)
$$

where by $\frac{d \sigma^{*}}{d p}\left(p=1, h_{2}\right)$ we mean the first derivative of $\sigma^{*}(p)$ for $G$ with two bond conductivities, 1 with probability $p$ and $h_{2}$ with probability $1-p$, evaluated at $p=1$. To evaluate $\sum_{1}$ in terms of $h_{2}$, we use (3.22), since for large $\beta, h_{2}$ is very close to 1 , so that $1 / s=1-h_{2}$ is very small. Using (5.22) gives

$$
\sum_{1}=\frac{\gamma_{2}}{\beta}-\frac{\gamma_{2}^{2}(1-1 / d)}{\beta^{2}}+O\left(\frac{1}{\beta^{3}}\right)
$$

Now we consider $\sum_{2}$. In view of (3.26) and Lemma 3.4,

$$
\sum_{2}=\frac{\partial^{2} \sigma^{*}}{\partial q_{1} \partial q_{2}}\left(p=1, h_{1}, h_{2}\right),
$$

where the right side of (5.61) refers to the mixed partial of the bulk conductivity of $G$ with bond conductivities $h_{1}=0$ with probability $q_{1}, h_{2}$ with probability $q_{2}$, and 1 with probability $p=1-q_{1}-q_{2}$, evaluated at $p=1$. Now for $s_{1}>1$ and $s_{2}>1$, Theorem 3.5 provides a convergent expansion for $\frac{\partial^{2} \sigma^{*}}{\partial q_{1} \partial q_{2}}$. Assuming $\frac{\partial^{2} \sigma^{*}}{\partial q_{1} \partial q_{2}}$ exists as, say, $s_{1} \rightarrow 1\left(\varepsilon_{1} \rightarrow 0\right)$, (3.47) still provides a convergent expression, although the first term is no longer necessarily the dominant one, so that we do not know the sign of the result. We can still write

$$
\sum_{2}=\frac{1}{s_{2}}\left(e_{0}+\frac{e_{1}}{s_{2}}+\frac{e_{2}}{s_{2}^{2}}+\ldots\right), \quad \frac{1}{s_{2}}=1-h_{2}
$$

for some set of constants $e_{j}, j=0,1, \ldots$, for which (5.62) converges. Then

$$
\sum_{2}=\frac{e_{0} \gamma_{2}}{\beta}+O\left(\frac{1}{\beta^{2}}\right)
$$

Combining (5.60) and (5.63) gives

$$
\delta_{12}=\alpha_{N} \gamma_{2}\left(1+e_{0}\right) n_{1}+O\left(\frac{1}{\beta}\right) .
$$


In general, we have

$$
\delta_{1 k}=\frac{\alpha_{N} \gamma_{k}\left(1+e_{0}\right) n_{1}}{\beta^{k-2}}+O\left(\frac{1}{\beta^{k-1}}\right), \quad k \geqq 3 .
$$

Consider now $\delta_{22}$. The pairs with both bonds in one necklace can be divided into three types: (1) both bonds are on the same first order bead but are removed in parallel, (2) both bonds are on the same first order bead but are removed in series, and (3) the bonds are on different first order beads, and are therefore removed in series. The fourth type of pair is when both bonds are in different necklaces. For a fixed necklace, or pair of necklaces in type 4, since there are $\beta-1$ beads per necklace, the number of each type of pair is

$$
\begin{gathered}
(\beta-1)\left(\beta n_{2}\right)^{2}=(\beta-1) n_{1}^{2} / 4, \\
2(\beta-1) \beta n_{2}\left(\beta n_{2}-1\right)=\frac{\beta n_{1}^{2}}{2}-\beta n_{1}-\frac{n_{1}^{2}}{2}+n_{1}, \\
2(\beta-1) \beta n_{2} \cdot 2(\beta-2) \beta n_{2}=n_{1}^{2}\left(\beta^{2}-3 \beta+2\right),
\end{gathered}
$$

and

$$
\left(\beta n_{1}\right)^{2} \text {. }
$$

Now, making observations analogous to (5.57), and noting, for example, that the removal of both bonds from type 1 pairs amounts to replacing the corresponding bond of $G$ with a bond of conductivity $h_{1}=0$, we can write

$$
\begin{aligned}
\delta_{22}= & z_{0}\left[\left((\beta-1) n_{1}^{2} / 4\right) \sum_{i \in G}\left(\sigma_{i}^{*}(1)+\sigma_{i}^{*}\left(h_{1}\right)-2 \sigma_{i}^{*}\left(h_{2}\right)\right)\right. \\
& +\left(\frac{\beta n_{1}^{2}}{2}-\beta n_{1}-\frac{n_{1}^{2}}{2}+n_{1}\right) \sum_{i \in G}\left(\sigma_{i}^{*}(1)-\sigma_{i}^{*}\left(h_{2}\right)\right) \\
& +n_{1}^{2}\left(\beta^{2}-3 \beta+2\right) \sum_{i \in G}\left(\sigma_{i}^{*}(1)+\sigma_{i}^{*}\left(h_{22}^{s}\right)-2 \sigma_{i}\left(h_{2}\right)\right) \\
& \left.+\left(\beta n_{1}\right)^{2} \sum_{\substack{i, j \in G \\
i \neq j}}\left(\sigma_{i j}^{*}(1,1)+\sigma_{i j}^{*}\left(h_{2}, h_{2}\right)-\sigma_{i j}^{*}\left(1, h_{2}\right)-\sigma_{i j}^{*}\left(h_{2}, 1\right)\right)\right] .
\end{aligned}
$$

Let us again denote the four sums in order in (5.66) as $\sum_{1}, \sum_{2}, \sum_{3}$, and $\sum_{4}$. We can write $\sum_{1}$ as

$$
\begin{aligned}
\sum_{1} & =\sum_{i \in G}\left(\sigma_{i}^{*}(1)+\sigma_{i}^{*}(1)-\sigma_{i}^{*}(1)+\sigma_{i}^{*}(0)-2 \sigma_{i}^{*}\left(h_{2}\right)\right) \\
& =\sum_{i \in G} 2\left(\sigma_{i}^{*}(1)-\sigma_{i}^{*}\left(h_{2}\right)\right)-\left(\sigma_{i}^{*}(1)-\sigma_{i}^{*}(0)\right),
\end{aligned}
$$

so that

$$
\sum_{1}=2 \frac{d \sigma^{*}}{d p}\left(p=1, h_{2}\right)-\frac{d \sigma^{*}}{d p}\left(p=1, h_{1}=0\right)
$$

Using (5.47) and (3.22), we have

$$
\Sigma_{1}=-\frac{d}{d-1}+\frac{2 \gamma_{2}}{\beta}+O\left(\frac{1}{\beta^{2}}\right) .
$$

Note that $\sum_{1}$ is thus negative, since we are considering two bonds in parallel. 
For $\sum_{2}$, clearly we have from (3.22)

$$
\sum_{2}=\frac{\gamma_{2}}{\beta}+O\left(\frac{1}{\beta^{2}}\right) .
$$

Note that $\sum_{2}$ is positive, since we are considering two bonds in series.

For $\sum_{3}$, we have after some calculation

$$
\sum_{3}=\frac{\alpha_{N}^{2} / 4 \alpha_{N-1}^{2}-2 \gamma_{2}^{2}}{\beta^{2}}+O\left(\frac{1}{\beta^{3}}\right),
$$

which can be shown to be positive, as we are concerned here with two bonds in series. We also have

$$
\sum_{4}=\frac{2 \gamma_{2}^{2} / d}{\beta^{2}}+O\left(\frac{1}{\beta^{3}}\right) .
$$

Summing our contributions gives

$$
\delta_{22}=\frac{-\alpha_{N} d n_{1}}{4(d-1)}+O\left(\frac{n_{1}}{\beta}\right) .
$$

For general $\delta_{k k}$, as in (5.74), the dominant (negative) term will come from choosing two $k^{\text {th }}$ order string bonds in parallel, which will break a necklace of order $k-1$. The net effect will be to replace $h_{1}=0$ in the second term on the right in (5.69) by $h_{k-1}$ with a net effect,

$$
\delta_{k k}=O\left(\frac{n_{1}}{\beta^{k-2}}\right), \quad k \geqq 1 .
$$

We now calculate $\delta_{23}$, with the same ordering of pairs as in the calculation of $\delta_{22}$. We omit the calculation of the numbers of each type of pair. Analogous to $\delta_{22}$, we have,

$$
\begin{aligned}
\delta_{23}= & z_{0}\left[\frac{(\beta-1)^{2}}{\beta} n_{1}^{2} \sum_{i \in G}\left(\sigma_{i}^{*}(1)+\sigma_{i}^{*}\left(h_{23}^{p}\right)-\sigma_{i}^{*}\left(h_{2}\right)-\sigma_{i}^{*}\left(h_{3}\right)\right)\right. \\
& +\frac{(\beta-1)^{2}}{\beta} n_{1}^{2} \sum_{i \in G}\left(\sigma_{i}^{*}(1)-\sigma_{i}^{*}\left(h_{3}\right)\right) \\
& +\frac{(\beta-1)^{2}(\beta-2)}{\beta} n_{1}^{2} \sum_{i \in G}\left(\sigma_{i}^{*}(1)+\sigma_{i}^{*}\left(h_{23}^{s}\right)-\sigma_{i}^{*}\left(h_{2}\right)-\sigma_{i}^{*}\left(h_{3}\right)\right) \\
& \left.+\left(\beta n_{1}\right)^{2} \sum_{\substack{i, j \in G \\
i \neq j}}\left(\sigma_{i j}^{*}(1,1)+\sigma_{i j}^{*}\left(h_{2}, h_{3}\right)-\sigma_{i j}^{*}\left(1, h_{2}\right)-\sigma_{i j}^{*}\left(1, h_{3}\right)\right)\right] .
\end{aligned}
$$

With calculations similar to those above, we obtain

$$
\begin{aligned}
& \sum_{1}=\frac{\gamma_{3}-\gamma_{2} / 2}{\beta^{2}}+O\left(\frac{1}{\beta^{3}}\right), \\
& \sum_{2}=\frac{\gamma_{3}}{\beta^{2}}+O\left(\frac{1}{\beta^{3}}\right), \\
& \sum_{3}=\frac{\gamma_{2}^{2} / d}{\beta^{2}}+O\left(\frac{1}{\beta^{3}}\right) .
\end{aligned}
$$


For $\sum_{4}$ we use (3.47),

$$
\begin{aligned}
\sum_{4} & =\frac{\partial^{2} \sigma^{*}}{\partial q_{1} \partial q_{2}}\left(p=1, h_{2}, h_{3}\right) \\
& =\frac{2 \gamma_{2} \gamma_{3} / d}{\beta^{3}}+O\left(\frac{1}{\beta^{4}}\right)
\end{aligned}
$$

Thus

$$
\delta_{23}=\frac{\alpha_{N} \gamma_{2}^{2} n_{1} / d}{\beta}+O\left(\frac{n_{1}}{\beta^{2}}\right) .
$$

For general $\delta_{j k}, k>j$, we still have four sums corresponding to $\sum_{1}, \sum_{2}, \sum_{3}, \sum_{4}$ above. In each case the net contribution is controlled primarily by $k$. In the case of $\sum_{1}$ and $\sum_{3}$, this is because, as remarked previously, $h_{j k}^{s}$ and $h_{j k}^{p}$ both match $h_{j}$ to $O\left(1 / \beta^{j-1}\right)[\operatorname{see}(5.22),(5.44)$, and (5.45)], so that the dominant terms here come from the $O\left(\beta^{k-1}\right)$ terms in (5.44) and (5.45). However, if $k-1>2(j-1)$, then the contribution is $O\left(1 / \beta^{2(j-1)}\right)$. For $\sum_{2}, h_{k}$ replaces $h_{3}$ in (5.76). For $\sum_{4}$, the leading order contribution comes from a product of $\left(1-h_{j}\right)$ and $\left(1-h_{k}\right)$, as in $(5.80)$, which will be smaller than the other sums. Thus, in general, we have

$$
\delta_{j k}=O\left(\frac{n_{1}}{\beta^{k-2}}\right), \quad 1 \leqq j<k,
$$

unless $(k-1)>2(j-1)$, in which case

$$
\left|\delta_{j k}\right| \leqq O\left(n_{1} / \beta^{2 j-3}\right) .
$$

Finally, summing all the $\delta_{j k}$ yields (5.48).

Due to the structure of $z_{k}, z_{k k}^{s}, z_{k k}^{p}, z_{j k}^{s}$, and $z_{j k}^{p}$ and their associated $\gamma$ 's, we may take the limit in (5.48) as $N \rightarrow \infty$. This must be done in such a way that $n_{1} \gg N$, so that there is enough "room" to add more levels of the hierarchy to the necklace. We can take this limit because the $\gamma_{k}$ 's, $\gamma_{k k}^{s}$ 's, and so on, decrease to 0 geometrically fast, so that any infinite sum of such coefficients also converges. Furthermore, all the perturbation expansions we have used are just convergent geometric series as well. In the dominant term of (5.48), we have $\lim _{N \rightarrow \infty} \alpha_{N}=3 / 4$. We have thus proven the theorem.

Remark 1. Theorem 5.2 and its proof hold for a variety of base lattices $G$. The first necessary ingredient, is of course, the validity of Hypothesis 5.1, which holds in great generality, as discussed above. The second major ingredient to make the above proof go through is the representations for the derivatives of $\sigma^{*}(p)$ obtained in Sect. 3. For regular, periodic lattices such as the hexagonal or triangular, these representations are elementary to obtain. Adjusted coefficients of the perturbation expansions at and beyond second order (in $1 / s$ ) replace those for the hypercubic lattice. For example, with $G$ the hexagonal lattice in $d=2$, an easy calculation shows that the coefficient of $1 / s^{2}$ in $(3.20)$ becomes $p(1-p) / 3$, which does not affect the validity of our proof. Presumably such calculations can be carried out for certain random or quasiperiodic lattices, and our asymptotics should hold for them as well.

Remark 2. The particular structure of the necklaces that we have used was chosen because it reflects what is known about the backbone structure, and it was 
relatively easy to compute exactly their conductivities with various bonds removed. However, similar results to (5.48) can be obtained with more complex necklace structures. For example, (5.11) could be replaced with $n_{j-1}=\eta_{j} \beta_{j} n_{j}$, with reasonable assumptions on $\eta_{j}$ and $\beta_{j}$, and results similar to (5.48) would be obtained. The blobs themselves could also be assumed to have a more complicated "super-lattice" structure, reflecting the macroscopic structure on the scale of $\xi$, and again similar results would be obtained. Finally, while our principal assumption of the NLB graph replacing the actual backbone is quite serious, our proof of (5.48) shows that the dominant contribution comes from $\delta_{11}$, which comes from macroscopic contributions in the NLB graph, where the model reflects well the actual structure. A similar result should hold for any reasonable assumption about microscopic backbone structure.

We now proceed to the implications of Theorem 5.2. Our first observation is that $\delta^{2} \sigma^{*}\left(A\left(n_{1}\right)\right)$ is positive, but the scaling provided by (5.50) yields more than just this convexity. The length $\lambda\left(n_{1}\right)$ of a $1^{\text {st }}$ order necklace which is "stretched out" is given by

$$
\lambda\left(n_{1}\right)=\beta n_{1}+\beta^{2} n_{2}+\ldots+\beta^{N} n_{N} .
$$

Using (5.12), we have

with $\lim _{N \rightarrow \infty} \theta_{N}=2$.

$$
\lambda\left(n_{1}\right)=\theta_{N} \beta n_{1}, \quad \theta_{N}=\sum_{i=0}^{N}\left(\frac{1}{2}\right)^{i}
$$

Thus, there is a constant $\varrho_{N}=\alpha_{N} \kappa(G) / \theta_{N}$ such that

$$
\delta^{2} \sigma^{*}\left(A\left(n_{1}\right)\right) \sim \frac{\varrho_{N} \lambda\left(n_{1}\right)}{\xi^{d-2}}, \quad n_{1}, \beta, N, \xi \rightarrow \infty,
$$

where in $d=2$ with large $N, \varrho_{N} \approx 2 / 3$. Now since all the parameters are diverging as $p \rightarrow p_{c}^{+}$, we can define a whole class of NLB models by how fast $\lambda\left(n_{1}\right) \rightarrow \infty$ as compared to $\xi \rightarrow \infty$. By the structure of the model, we must have

$$
\lambda\left(n_{1}\right) \geqq \xi \text {. }
$$

Since $\lambda$ and $\xi$ are both diverging, $\varrho_{N}$ does not affect the gross asymptotic behavior of $\delta^{2} \sigma^{*}\left(A\left(n_{1}\right)\right)$. Thus, in view of (5.86) and (5.50), we have

Corollary 5.1. Let $A\left(n_{1}\right)$ be the NLB graph formed from the hypercubic base lattice $G$, which is assumed to satisfy the conditions in Theorem 5.2. Then in $d=2,3$,

$$
\delta^{2} \sigma^{*}\left(A\left(n_{1}\right)\right) \rightarrow+\infty, \quad n_{1}, \beta, N, \xi \rightarrow \infty,
$$

except in $d=3$, when $\lambda\left(n_{1}\right)=C \xi, C \geqq 1$, in which case

$$
\delta^{2} \sigma^{*}\left(A\left(n_{1}\right)\right) \rightarrow \varrho C>0, \quad n_{1}, \beta, N, \xi \rightarrow \infty,
$$

where $\varrho=\lim _{N \rightarrow \infty} \varrho_{N}$. In $d \geqq 4$, if $\lambda$ and $\xi$ are scaled so that

$$
\frac{\lambda\left(n_{1}\right)}{\xi^{d-2}} \rightarrow 0^{+}, \quad n_{1}, \beta, N, \xi \rightarrow \infty,
$$

then

$$
\delta^{2} \sigma^{*}\left(A\left(n_{1}\right)\right) \rightarrow 0^{+}, \quad n_{1}, \beta, N, \xi \rightarrow \infty .
$$

Before we relate these results back to the actual problem to give exponent inequalities, let us briefly consider the third derivative $\frac{d^{3} \sigma^{*}}{d p^{3}}$. We shall only be using 
this derivative for information in $d \geqq 4$. In this case the blobs become somewhat less relevant [52], and we consider here only the node-link model, which is formed by replacing the bonds of $G$ with $\lambda(n)=n$ bonds in series, and the nodes are still separated by $\xi$. For the analog $\delta^{3} \sigma$ of $\delta^{2} \sigma$, we have the analog of Theorem 5.1,

Theorem 5.3. Let $G$ be any finite graph with unit bond conductivities, and let $S_{n} G$ be a new graph formed by replacing each bond of $G$ with $n$ bonds in series. Then there exists a constant $C_{3}$ such that

$$
\delta^{3} \sigma\left(S_{n} G\right) \sim C_{3} n^{2}, \quad n \rightarrow \infty .
$$

Proof. Differentiate (5.6) three times.

We then have

Corollary 5.2. Let $A^{\prime}(n)$ be the node-link graph formed from the hypercubic base lattice $G$. Then

$$
\delta^{3} \sigma^{*}\left(A^{\prime}(n)\right) \sim \frac{C_{3} n^{2}}{\xi^{d-2}}
$$

so that for models in $d=4,5$ with

$$
\frac{[\lambda(n)]^{2}}{\xi^{d-2}} \rightarrow+\infty
$$

we have

$$
\delta^{3} \sigma^{*}\left(A^{\prime}(n)\right) \rightarrow \infty, \quad n, \xi \rightarrow \infty .
$$

Now we relate Corollaries 5.1 and 5.2 back to the actual problem.

Corollary 5.3. Let $\sigma^{*}(p)$ be the bulk conductivity of the hypercubic lattice with bond conductivities 1 and 0 in proportions $p$ and $1-p$. Assuming $\sigma^{*}(p) \sim\left(p-p_{c}\right)^{t}$ as $p \rightarrow p_{c}^{+}$, and that it has at least three derivatives for all $p \in\left(p_{c}, 1\right]$, and assuming that the actual backbone $B(p)$ in (3.16) is replaced by the NLB graph $A\left(n_{1}\right)$ for $p$ near $p_{c}$ with the hypercubic base lattice $G$ satisfying the conditions in Theorem 5.2, then

$$
\begin{gathered}
1 \leqq t \leqq 2, \quad d=2,3, \\
2 \leqq t \leqq 3, \quad d \geqq 4,
\end{gathered}
$$

for models which satisfy (5.90) and (5.94) for $d=4,5$, where in (5.97) the inequality $t \leqq 3$ is obtained by replacing $B(p)$ by the node-link graph $A^{\prime}(n)$.

Acknowledgements. It is a pleasure to thank a number of people for helpful discussions during the course of the work: D. Bergman, O. Bruno, J. Chayes, L. Chayes, D. Fisher, H. Kesten, C. Lobb, and S. Varadhan. Special thanks is owed to P. Doyle for many long and useful discussions during the early stages of the work, and to G. Papanicolaou for his constant encouragement and interest in this work, as well as helpful discussions. D. Barsky provided many useful comments which significantly streamlined the original manuscript, which is gratefully acknowledged.

\section{References}

1. Ziman, J.M.: J. Phys. C 1, 1532 (1968)

2. Eggarter, T.P., Cohen, M.H.: Phys. Rev. Lett. 25, 807 (1970)

3. Eggarter, T.P., Cohen, M.H.: Phys. Rev. Lett. 27, 129 (1971)

4. Broadbent, S.R., Hammersly, J.M.: Proc. Cambridge Philos. Soc. 53, 629 (1957)

5. Last, B.J., Thouless, D.J.: Phys. Rev. Lett. 27, 1719 (1971) 
6. Kirkpatrick, S.: Phys. Rev. Lett. 27, 1722 (1971)

7. Stauffer, D.: Introduction to percolation theory. London: Taylor and Francis 1985

8. Kesten, H.: Percolation theory for mathematicians. Boston: Birkhäuser 1982

9. Grimmett, G.: Percolation. Berlin, Heidelberg, New York: Springer 1989

10. Kirkpatrick, S.: Rev. Mod. Phys. 45, 574 (1973)

11. Stauffer, D.: Phys. Rep. 54, 1 (1979)

12. Grimmett, G., Kesten, H.: Z. Wahr. 66, 335 (1984)

13. Chayes, J.T., Chayes, L.: Commun. Math. Phys. 105, 133 (1986)

14. Kozlov, S.M.: Russ. Math. Surv. 44, 91 (1989)

15. Straley, J.P.: Phys. Rev. C 15, 2333 (1982)

16. Chayes, J., Chayes, L., Kesten, H.: Unpublished

17. Watson, B.P., Leath, P.L.: Phys. Rev. B 9, 4893 (1974)

18. Harris, A.B., Kirkpatrick, S.: Phys. Rev. B 16, 542 (1977)

19. Ernst, M.H., van Velthoven, P.F.J., Nieuwenhuizen, Th.M.: J. Phys. A 20, 949 (1987)

20. Nieuwenhuizen, Th.M., van Velthoven, P.F.J., Ernst, M.H.: Phys. Rev. Lett. 57, 2477 (1986)

21. Golden, K.: In: Random media and composites. Kohn, R.V., Milton, G.W. (eds.) Philadelphia: SIAM 1989

22. Bruno, O.: In preparation

23. Stanley, H.E.: J. Phys. A 10, L211 (1977)

24. Coniglio, A.: J. Phys. A 15, 3829 (1982)

25. Gingold, D.B., Lobb, C.J.: Phys. Reve. B 42, 8220 (1990)

26. Adler, J., Meir, Y., Aharony, A., Harris, A.B., Klein, L.: J. Stat. Phys. 58, 511 (1990)

27. Harris, A.B., Kim, S., Lubensky, T.C.: Phys. Rev. Lett. 53, 743 (1984)

28. Roman, H.E.: J. Stat. Phys. 58, 375 (1990)

29. Golden, K: Phys. Rev. Lett. 65, 2923 (1990)

30. Kozlov, S.M.: Dokl. Akad. Nauk. SSSR 241, 1016 (1978)

31. Papanicolaou, G., Varadhan, S.: Colloquia mathematica sociatatis János Bolyai, vol. 27. Random Fields. Amsterdam: North-Holland 1982

32. Golden, K., Papanicolaou, G.: Commun. Math. Phys. 90, 473 (1983)

33. Künnemann, R.: Effective conductivity on a lattice as a limit of box conductivities. In: Ph.D. Thesis, U. of Heidelberg (1983)

34. Zhikov, V.V.: Math. Zametki 45, 34 (1989)

35. Bergman, D.J.: Phys. Rep. C 43, 377 (1978)

36. Akhiezer, N.I., Glazman, I.M.: The theory of linear operators in Hilbert space. New York: Ungar 1966

37. Lamperti, J.: Probability. Reading, MA: Benjamin 1966

38. Bruno, O.: The effective conductivity of an infinitely interchangeable mixture. Ph.D. Thesis, New York University 1989

39. Miller, M.N.: J. Math. Phys. 10, 1988 (1969)

40. Bruno, O., Golden, K.: J. Stat. Phys. 61, 361 (1990)

41. Golden, K.: Bulk conductivity of the square lattice for complex volume fraction. To appear in the Proceedings of the Oberwolfach Conference on Random Partial Differential Equations. Hornung, U., Kotelemez, P., Papanicolaou, G. (eds.) Boston: Birkhäuser 1991

42. Golden, K., Papanicolaou, G.: J. Stat. Phys. 40, 655 (1985)

43. Straley, J.P.: Phys. Rev. B 15, 5733 (1977)

44. Keller, J.B.: J. Math. Phys. 5, 548 (1964)

45. Dykhne, A.M.: Sov. Phys. JETP 32, 63 (1971)

46. Sykes, M.F., Essam, J.W.: J. Math. Phys. 5, 1117 (1964)

47. Chayes, J.T., Chayes, L., Grimmett, G.R., Kesten, H., Schonmann, R.H.: Ann. Prob. 17, 1277 (1989)

48. Skal, A.S., Shlovskii, B.I.: Sov. Phys.-Semicond. 8, 1029 (1975)

49. de Gennes, P.G.: J. Physique Lett. 37, L1 (1976)

50. Stinchcombe, R.B.: J. Phys. C6, L1 (1973)

51. Straley, J.P.: J. Phys. C 10, 3009 (1977)

52. Harris, A.B.: Phys. Rev. B 28, 2614 (1983) 
